\title{
THE APPROXIMATION OF HARMONIC FUNCTIONS BY HARMONIC POLYNOMIALS AND BY HARMONIC RATIONAL FUNCTIONS*
}

BY J. L. WALSH

1. Introduction. The following theorem of Weierstrass is classical.

Let the function $f(\theta)$ be continuous for all values of the argument and periodic with period $2 \pi$. Then if an arbitrary positive $\epsilon$ be given, there exists a trigonometric polynomial which differs from $f(\theta)$ at most by $\epsilon$; that is, the inequality

$$
\left|f(\theta)-\sum_{k=0}^{n}\left(a_{k} \cos k \theta+b_{k} \sin k \theta\right)\right| \leqq \epsilon
$$

holds for all values of $\theta$.

An equivalent statement of the conclusion of this theorem is that $f(\theta)$ can be expanded in a series of the form

$$
\sum_{n=0}^{\infty} \sum_{k=0}^{n}\left(a_{n k} \cos k \theta+b_{n k} \sin k \theta\right),
$$

which converges uniformly for all values of $\theta$. This is of course a general fact, that if a given function can be uniformly approximated as closely as desired by a linear combination of other functions, then that function can be expanded in a uniformly convergent series of which each term is a linear combination of those other functions, and conversely. This fact is easy to prove and will be frequently used in the sequel.

If in the $(x, y)$-plane we introduce polar coordinates $(r, \theta)$ and consider the function $f(\theta)$ defined on the unit circle $C$, Weierstrass's theorem refers to the approximation of a function $f(\theta)$ continuous on $C$ by trigonometric polynomials, or what is the same thing, by polynomials of the form

${ }^{*}$ An address delivered by invitation of the program committee at the meeting of the Society in New York, February 23, 1929. 


$$
\sum_{k=0}^{n} r^{k}\left(a_{k} \cos k \theta+b_{k} \sin k \theta\right) \text {. }
$$

A polynomial of the form (2) is of course harmonic, for the expressions $r^{k} \cos k \theta$ and $r^{k} \sin k \theta$ are respectively the real parts of the analytic functions $z^{k},-i z^{k}$, and can be written as harmonic polynomials in $x$ and $y$.

If we interpret Weierstrass's theorem as yielding a uniform expansion rather than uniform approximation of $f(\theta)$, we have a sequence $\left\{p_{n}(r, \theta)\right\}$ of functions each of type (2) which converges uniformly to the function $f(\theta)$ on $C$ and likewise converges uniformly in the closed interior of $C$. For a necessary and sufficient condition for uniform convergence on $C$ is that if an arbitrary $\epsilon$ be given, there exist $M$ so that

$$
\left|p_{m}(r, \theta)-p_{n}(r, \theta)\right| \leqq \epsilon, \quad m, n>M,
$$

holds on $C$. But this inequality holding for $r=1$ holds also for $r \leqq 1$, since the functions $p_{n}(r, \theta)$ are harmonic for $r \leqq 1$ and a harmonic function has its maximum and minimum values on the boundary of the region considered. The limit $f(r, \theta)$ of this sequence $\left\{p_{n}(r, \theta)\right\}$ is thus harmonic* interior to $C$, continuous in the corresponding closed region, and on $C$ coincides with the given continuous function $f(\theta)$. That is, by Weierstrass's theorem we have established the existence of the function $f(r, \theta)$, the solution of the Dirichlet problem for the region interior to $C$ and for arbitrary continuous boundary values $f(\theta)$; the solution of the Dirichlet problem is known to be unique.

The problem of the uniform approximation to $f(\theta)$ on $C$ by harmonic polynomials is precisely equivalent to the problem of the uniform approximation to $f(r, \theta)$ in the closed interior of $C$, for a function harmonic in a region has. no maximum or minimum interior to that region. Inequality (1) is equivalent to the inequality

* A function $u(x, y)$ is harmonic in a region if it is continuous there, together with its first and second partial derivatives, and if $\left(\partial^{2} u / \partial x^{2}\right)+$ $\left(\partial^{2} u / \partial y^{2}\right)=0$. A function is harmonic at a point if it is harmonic throughout a neighborhood of that point. 


$$
\left|f(r, \theta)-\sum_{k=0}^{n} r^{k}\left(a_{k} \cos k \theta+b_{k} \sin k \theta\right)\right| \leqq \epsilon, \quad r \leqq 1 ;
$$

each of these inequalities implies the other.

The discussion just given suggests the general problem of studying the approximation to a given continuous function on an arbitrary point set $C$ by means of harmonic polynomials or more generally by harmonic rational functions, particularly in the more interesting cases that the given function is harmonic in the interior points of $C$, or that $C$ is itself the boundary of a region. The present paper is devoted to results on this general problem, chiefly a report so far as concerns approximation by polynomials, but a detailed exposition of results on approximation by more general rational functions, for these results are in the main new. As in the special case already considered to some extent, namely the theory of approximation to functions of a single real variable by trigonometric polynomials, there are particularly three special problems to be taken up here: first, the general problem of the possibility of approximation, corresponding to Weierstrass's theorem as already mentioned; second, the problem of approximation by special types of polynomials corresponding for example to expansion in Fourier's series; and third, the study of the degree of approximation, the study of the relation between the continuity properties of the functions approximated and the least maximum error for approximation by a polynomial of degree $n$. We shall examine these problems in some detail in the order given, first the problems for approximation by polynomials and later the corresponding problems for approximation by more general rational functions.

In the theory of functions of the complex variable $z=x+i y$, similar problems arise in connection with the approximating on a given point set of a given continuous or analytic function by means of polynomials in $z$ or rational functions of $z$. This analogous theory frequently suggests results in the present theory of harmonic functions, and 
may even furnish methods of proving those results; but the connection between the two theories is not always simple, and some situations in each theory have no analogs in the other. The two theories both have interesting applications to and connections with harmonic and analytic continuation, conformal mapping, the study of the Dirichlet problem, and topology.

2. General Approximation by Harmonic Polynomials. The fundamental theorem in the approximation of analytic functions by polynomials is due to Runge [1]*:

Let $f(z)$ be a function of $z$, analytic in a simply-connected region $\dagger C$ of the z-plane which does not contain the point at infinity in its interior. Then $f(z)$ can be developed in $C$ in a series of polynomials in $z$, which converges uniformly on any closed point set interior to $C$.

We shall use Runge's theorem to prove the following ana$\log$ for harmonic functions.t

Let $u(x, y)$ be a function of $(x, y)$, harmonic in a simplyconnected region $C$ of the $(x, y)$-plane which does not contain the point at infinity in its interior. Then $u(x, y)$ can be developed in $C$ in a series of harmonic polynomials in $(x, y)$, which converges uniformly on any closed point set interior to $C$.

Let $v(x, y)$ be a function conjugate to $u(x, y)$ in $C$; the function $v(x, y)$ is single-valued in $C$ if one considers only an element of $v(x, y)$ and its harmonic extensions along curves interior to $C$. Runge's theorem as applied to the function $f(z)=u(x, y)+i v(x, y)$ yields a development of $f(z)$ in polynomials in $z$. The series whose terms are the real parts of the respective terms of that development represents the function $u(x, y)$ and has the required properties with respect to convergence.

* The numbers in square brackets refer to the works of the authors indicated, in the bibliography at the close of this paper.

$\dagger$ A region is a connected set of interior points. A region plus its boundary points forms the corresponding closed region.

$\ddagger$ Compare Walsh [1, p. 198]. Bergmann [1] uses similar reasoning, but does not bring out clearly the uniformity of the convergence of the series of harmonic polynomials. 
The essential content for our present purposes of the theorem just proved may also be stated as follows.

If the function $u(x, y)$ is harmonic in a closed Jordan region, then $u(x, y)$ can be developed in that closed region in a uniformly convergent series of harmonic polynomials in $(x, y)$.

The present writer has proved, however, results much more general than this [1], regarding the uniform development of arbitrary harmonic functions. We state a number of these results, simply mentioning at this time the fact that the proof is based on Lebesgue's important work [1] on harmonic functions. We shall go into more detail regarding methods in $\$ 5$, when we take up the more general problem of development in terms of harmonic rational functions.

Let $C$ be an arbitrary limited simply-connected region of the $(x, y)$-plane. $A$ necessary and sufficient condition that an arbitrary function harmonic in $C$, continuous in the corresponding closed region, be uniformly developable in the closed region by harmonic polynomials in $(x, y)$, is that the boundary of $C$ be also the boundary of an infinite region.

Let $C$ be an arbitrary limited closed point set. A necessary and sufficient condition that an arbitrary function continuous on $C$ be uniformly developable on $C$ by harmonic polynomials in $(x, y)$, is that $C$ be the boundary of an infinite region.

An arbitrary limited closed point set $C$ divides the plane in general into a number of regions; in particular one of these, $D$, is infinite. A continuous function $u(x, y)$ defined on $C$ is in particular defined on the boundary $B$ of $D$, and on this boundary can, by the theorem just stated, be uniformly developed in harmonic polynomials. This expansion of $u(x, y)$ on $B$ converges uniformly on $B$, hence uniformly on the entire point set $E$ which is complementary (with respect to the entire plane) to $D$, and therefore defines a function $U(x, y)$ on the entire set $E$. The set $C$ is a subset of $E$. The functions $u(x, y)$ and $U(x, y)$ coincide on $B$. A necessary and sufficient condition that $u(x, y)$ be uniformly developable on $C$ in harmonic polynomials is that $u(x, y)$ and $U(x, y)$ should coincide on $C$. 
These theorems obviously include Weierstrass's theorem on the approximation to an arbitrary continuous function by trigonometric polynomials. These theorems include also Weierstrass's classical theorem that an arbitrary function continuous on a closed interval of the axis of reals can be uniformly approximated on that closed interval as closely as desired by a polynomial in the real variable. For if the point set $C$ is chosen as such an interval, the only harmonic polynomials $1, r \cos \theta, r \sin \theta, \cdots, r^{n} \cos n \theta, r^{n} \sin n \theta, \cdots \operatorname{not}$ vanishing identically on $C$ can be written on $C$ in the form $1, x, x^{2}, \cdots$, so that on $C$ a harmonic polynomial is a polynomial in $x$.

With these results, the most important questions regarding the expansion of arbitrary functions on limited point sets are answered. The corresponding questions for unlimited point sets have apparently not been treated previously. On some unlimited point sets $C$ only the polynomial 1 of the set $\left\{r^{n} \cos n \theta, r^{n} \sin n \theta\right\}$ is uniformly bounded, so the only functions continuous on $C$ which can be uniformly approximated on $C$ by harmonic polynomials are constants. On certain other unlimited point sets $C$ only a finite number of the harmonic polynomials $\left\{r^{n} \cos n \theta, r^{n} \sin n \theta\right\}$ are continuous, and a necessary and sufficient condition that a function continuous on such a point set $C$ be uniformly developable on $C$ by harmonic polynomials is that the function should be a linear combination of those particular harmonic polynomials. On other unlimited point sets $C$ an infinity of the harmonic polynomials $\left\{r^{n} \cos n \theta, r^{n} \sin n \theta\right\}$ are continuous, so necessary and sufficient conditions for the expansion of arbitrary functions on such point sets $C$ are not obvious, nor can they be obtained without modification of the methods hitherto employed.

It is illuminating to contrast the present problem with the problem of approximating given analytic or continuous functions by means of polynomials in the complex variable. It is not yet known what is the most general region $C$ such that an 
arbitrary function analytic interior to $C$ and continuous in the corresponding closed region can be approximated uniformly in the closed region as closely as desired by polymials in the complex variable. It can be shown by an example [Walsh, 1, p. 203] however, that this most general region $C$ is not the most general region such that an arbitrary function harmonic interior to $C$ and continuous in the corresponding closed region can be approximated uniformly in the closed region as closely as desired by harmonic polynomials. Unlimited point sets $C$ are easy to treat for approximation by polynomials in the complex variable. Every such polynomial not a constant becomes infinite on $C$, so the only continuous functions which can be uniformly approximated on $C$ are constants, and every such function can be so approximated.

3. Harmonic Polynomials Belonging to a Region. It is natural to inquire whether more precise results than those just considered are obtainable regarding expansions in a given region in terms of a particular set of harmonic polynomials belonging to that region. For instance, if the region is chosen as the interior of the unit circle $C$, then an arbitrary function $f(x, y)$ harmonic in the closed interior of $C$ can be expanded on the circumference in a series of Fourier:

(3) $f(x, y)=\frac{a_{0}}{2}+\sum_{k=1}^{\infty}\left(a_{k} \cos k \theta+b_{k} \sin k \theta\right)$,

$$
a_{k}=\frac{1}{\pi} \int_{0}^{2 \pi} f \cos k \theta d \theta, b_{k}=\frac{1}{\pi} \int_{0}^{2 \pi} f \sin k \theta d \theta
$$

where the integrals are computed over $C$. The series converges uniformly on $C$. The series

$$
\frac{a_{0}}{2}+\sum_{k=1}^{\infty} r^{k}\left(a_{k} \cos k \theta+b_{k} \sin k \theta\right)
$$

is a series of harmonic polynomials which converges uni- 
formly on $C$ and hence converges uniformly throughout the closed interior of $C$. The sum of the series (4) coincides with $f(x, y)$ on $C$ and hence throughout the interior of $C$ as well.

If we start not with the unit circle but a more general Jordan curve $C$ as the boundary of our region, can there be found harmonic polynomials $\left\{p_{k}(x, y)\right\}$ such that an arbitrary function harmonic on and within $C$ can be expanded in a series

$$
\sum_{k=0}^{\infty} a_{k} p_{k}(x, y)
$$

which converges uniformly in the closed interior of $C$ ? The answer here is affirmative, if the curve $C$ is analytic. In fact, Faber [1], Fejér [1], Szegö [1], Carleman [1], Bergmann [1], Bochner [1], and others have studied polynomials $\left\{s_{k}(z)\right\}$ in the complex variable $z$ belonging to such a region and have shown that an arbitrary function $f(z)$ analytic in the closed region can be expanded in a series

$$
\sum_{k=0}^{\infty} c_{k} s_{k}(z)
$$

which converges uniformly in the closed region. A suitable set of harmonic polynomials of the kind we desire can be found by separating $s_{k}(z)$ into its real and pure imaginary parts. Thus, let the function $u(x, y)$ be given harmonic in the closed interior of $C$; there exists a function $v(x, y)$ conjugate to it, likewise harmonic in this closed region. Then by the properties of the polynomials $s_{k}(z)$, there exists an expansion

$u(x, y)+i v(x, y)=\sum_{k=0}^{\infty}\left(c_{k}^{\prime}+i c_{k}^{\prime \prime}\right)\left(s_{k}^{\prime}(x, y)+i s_{k}^{\prime \prime}(x, y)\right)$,

where $c_{k}=c_{k}^{\prime}+i c_{k^{\prime \prime}}, s_{k}(z)=s_{k}^{\prime}(x, y)+s_{k}^{\prime \prime}(x, y)$. From this we derive the series

$$
u(x, y)=\sum_{k=0}^{\infty}\left[c_{k}^{\prime} s_{k}^{\prime}(x, y)-c_{k}^{\prime \prime} s_{k}^{\prime \prime}(x, y)\right],
$$


so we have an expansion of type (5)* by setting

$$
p_{2 k}(x, y)=s_{k}^{\prime}(x, y), \quad p_{2 k+1}(x, y)=s_{k}^{\prime \prime}(x, y) .
$$

In particular if the derivatives of the polynomials $s_{k}(z)$ are orthogonal with respect to the area interior to $C$, the formulas for the coefficients in (6) are remarkably simple in form, as Bergmann has shown [1].

There are, however, various other ways of determining sets of harmonic polynomials belonging to a given region. Let us consider the plane of the auxiliary variables $\left(x^{\prime}, y^{\prime}\right)$, and map conformally the interior of $C$ onto the interior of the unit circle $\Gamma$ of the $\left(x^{\prime}, y^{\prime}\right)$-plane. A function harmonic in the closed interior of $C$ corresponds to a function harmonic in the closed interior of $\Gamma$, and can be uniformly expanded in the closed interior of $\Gamma$ in a series (4) of harmonic polynomials in $\left(x^{\prime}, y^{\prime}\right)$. The harmonic polynomials $r^{n} \cos n \theta, r^{n} \sin n \theta$ in the $\left(x^{\prime}, y^{\prime}\right)$-plane correspond in the $(x, y)$-plane to functions harmonic in the closed interior of $C$; moreover these functions in the $(x, y)$-plane can be replaced by harmonic polynomials in $(x, y)$ which differ only slightly from them, without essentially altering the convergence properties of series expansions of arbitrary functions in terms of them. More explicitly, we state the following theorem [Walsh, 2].

Let $C$ be a simple finite analytic curve in the $(x, y)$-plane. Then there exist harmonic polynomials $\left\{p_{k}(x, y)\right\}$ such that if $f(x, y)$ is defined and continuous on $C$ and on $C$ is of bounded variation, then $f(x, y)$ can be developed into a series

$$
f(x, y)=\sum_{k=0}^{\infty} a_{k} p_{k}(x, y),
$$

which converges uniformly in the closed interior of $C$. Series (7) thus represents a function harmonic interior to $C$, continuous in the corresponding closed region, and having the value $f(x, y)$ on $C$. There exist continuous functions $\left\{q_{k}(x, y)\right\}$ on $C$

* Removal of brackets in series (6) can be justified in the cases to which reference has been made. 
with which the polynomials $\left\{p_{k}(x, y)\right\}$ form a biorthogonal set:

$$
\int_{C} p_{k}(x, y) q_{m}(x, y) d s=\left\{\begin{array}{l}
0, \text { if } k \neq m, \\
1, \text { if } k=m .
\end{array}\right.
$$

The coefficients of (7) are given by the formulas

$$
a_{k}=\int_{C} f(x, y) q_{k}(x, y) d s
$$

the functions $q_{k}(x, y)$ depend on $C$ but not on $f(x, y)$.

If the function $f(x, y)$ is known merely to be continuous on $C$, then the series (7), where the $a_{k}$ are given by (8), converges throughout the interior of $C$, uniformly on any closed point set interior to $C$; if summed by the method of Cesàro this series converges uniformly on and within $C$ and thus represents a solution of the Dirichlet problem for the region interior to $C$ and the boundary values $f(x, y)$.

If the function $f(x, y)$ is of bounded variation on $C$, but not necessarily continuous, then the series (7), where the $a_{k}$ are given by (8), converges at every point of the closed region, uniformly on any closed point set interior to $C$. The function represented is bounded in the closed interior of $C$ and approaches the boundary values $f(x, y)$ continuously at every point of continuity of $f(x, y)$.

This theorem as stated applies only to an analytic curve $C$, but the writer has some further results, as yet unpublished, which apply to much more general curves.

Still another method of defining a set of harmonic polynomials belonging to a given region bounded by a rectifiable Jordan curve is that of orthogonalization. We begin with the harmonic polynomials

$$
1, r \cos \theta, r \sin \theta, r^{2} \cos 2 \theta, r^{2} \sin 2 \theta, \cdots
$$

and orthogonalize them with respect to the given curve $C$. This yields a set of polynomials $\left\{p_{k}(x, y)\right\}$ such that

$$
\int_{C} p_{k}(x, y) p_{m}(x, y) d s=\left\{\begin{array}{l}
0, k \neq m, \\
1, k=m .
\end{array}\right.
$$


An arbitrary function $f(x, y)$ defined on $C$ can be expanded formally in terms of these polynomials :

$$
f(x, y) \sim \sum_{k=0}^{\infty} a_{k} p_{k}(x, y), \quad a_{k}=\int_{C} f(x, y) p_{k}(x, y) d s .
$$

This orthogonalization method was used by Szegö [1] in the corresponding case of polynomials in the complex variable. The results in the present case have been established by Merriman [1], who determines the asymptotic formulas for the polynomials $\left\{p_{k}(x, y)\right\}$, and shows, under suitable restrictions, that the series (9) converges interior to $C$ or even uniformly in the closed interior of $C$. The sum of the series is of course the solution of the Dirichlet problem for the continuous boundary values $f(x, y)$.

lt would be of interest to investigate the corresponding problems where the harmonic polynomials are orthogonalized not with respect to arc length on the boundary, but with respect to the area of the region interior to $C$ [compare Bergmann 1, Carleman 1]. The former method has the theoretical advantage, however, of not requiring for expansion the knowledge of the values of the harmonic function except on the boundary of the region. Another general problem which deserves treatment is the detailed study of the convergence on $C$ of expansions in terms of the special polynomials of Faber and others, either for the case of analytic or of harmonic functions, where the given function to be expanded is not known to be analytic or harmonic in the closed region.

4. Degree of Approximation. We have considered thus far the possibility of approximation by harmonic polynomials and the expansion in terms of a particular set of harmonic polynomials. We turn now to consideration of the degree of approximation, that is, the relation between the properties of the function approximated, with regard to existence of derivatives etc. on the one hand, and the asymptotic properties of the maximum error in the best approximation 
by a harmonic polynomial of degree $n,{ }^{*}$ on the other hand. A simple result [Walsh, 3] here is the analog and consequence of the corresponding result in approximation of analytic functions by means of polynomials in the complex variable.

Let $C$ be an arbitrary closed Jordan region of the $(x, y)$-plane, and let $w=\phi(z), z=x+i y$, be a function which maps conformally the exterior of $C$ onto the exterior of the unit circle in the w-plane so that the points at infinity correspond to each other. Let $C_{R}$ denote the curve $|\phi(z)|=R, R>1$, that is, the transform in the z-plane of the circle $|w|=R$.

$A$ necessary and sufficient condition that an arbitrary function $u(x, y)$, defined in $C$, be harmonic in the (closed) region $C$ is that there should exist harmonic polynomials $p_{n}(x, y)$ of degree $n, n=0,1,2, \cdots$, and numbers $M, R>1$, such that the inequalities

$$
\left|u(x, y)-p_{n}(x, y)\right| \leqq \frac{M}{R^{n}},
$$

where $M$ and $R$ are independent of $n$ and of $(x, y)$, should be valid for every point $(x, y)$ of $C$.

If the polynomials $p_{n}(x, y)$ are given so that $(10)$ is satisfied for every $(x, y)$ of $C$, the sequence $\left\{p_{n}(x, y)\right\}$ converges everywhere interior to $C_{R}$ and uniformly on any closed point set interior to $C_{R}$, so the function $u(x, y)$ is harmonic throughout the interior of $C_{R}$. $\dagger$

If $u(x, y)$ is given harmonic in the closed region interior to $C_{\rho}$, the polynomials $p_{n}(x, y)$ can be chosen to satisfy (10) with $R=\rho$, for $(x, y)$ in $C$.

* The polynomial $a_{0} x^{n}+a_{1} x^{n-1} y+\cdots+a_{n} y^{n}+b_{0} x^{n-1}+b_{1} x^{n-2} y+\cdots$ is considered to be of degree $n$, but if the term degree is used in the restricted sense, is of degree $n$ if and only if at least one of the coefficients $a_{i}$ is different from zero.

$\dagger$ Here and below we tacitly assume that if $u(x, y)$ is not originally supposed to be defined on the entire point set considered, then the definition in the new points is to be made by harmonic extension-or what amounts to the same thing-by means of the convergent series of harmonic polynomials. 
This theorem seems to be the only one in the literature concerning degree of approximation by harmonic polynomials, except in the case that $C$ itself is a circle, when approximation by harmonic polynomials reduces to approximation on the circumference by trigonometric polynomials, for which well known results have been obtained by Bernstein, Jackson, Montel, de la Vallée-Poussin, and others [see de la Vallée-Poussin, 1]. There is obviously occasion here for further investigation. If $u(x, y)$ is given harmonic within, continuous on and within the Jordan curve $C$, what is the relation between the maximum error for the best approximation of $u(x, y)$ on $C$ by a harmonic polynomial of degree $n$ on the one hand, and the continuity properties of the curve $C$ and of the function $u(x, y)$ on $C$ on the other hand? If $u(x, y)$ is given harmonic within, continuous on and within $C_{R}$, what is the relation between the maximum error for the best approximation on $C$ by a harmonic polynomial of degree $n$ on the one hand, and the continuity properties of the curve $C$ and of the function $u(x, y)$ on the curve $C_{R}$ on the other hand?

Let us stop for a moment to consider the Tchebycheff harmonic polynomial for the function $u(x, y)$ on a point set $C$, that is, the harmonic polynomial $p_{n}(x, y)$ of degree $n$ for which the maximum $\left|u(x, y)-p_{n}(x, y)\right|,(x, y)$ on $C$, is least. It is convenient here to refer to a general theorem due to Haar [1], which deals with approximation on a given point set $C$ of a given function $u(x, y)$ by linear combinations of given functions $\left\{u_{i}(x, y)\right\}$; the coefficients $a_{i}$ are to be determined so that the maximum

$$
\left|u(x, y)-a_{1} u_{1}(x, y)-\cdots-a_{m} u_{m}(x, y)\right|
$$

is least for $(x, y)$ on $C$. If $C$ is closed and the $u$ 's are continuous, such a determination of the coefficients is possible [Haar, loc. cit.] and the corresponding linear combination of the $u_{i}(x, y)$ may be called a Tchebycheff polynomial of order $m$. Haar's theorem asserts-except for incidental restrictions on the continuity of the functions and the 
closure of the point set-that a necessary and sufficient condition for the uniqueness of the Tchebycheff polynomial of order $m$ for approximation on $C$ to an arbitrary function continuous on $C$, is that no function

$$
\begin{gathered}
A_{1} u_{1}(x, y)+\cdots+A_{m} u_{m}(x, y), \\
\left|A_{1}\right|+\left|A_{2}\right|+\cdots+\left|A_{m}\right| \neq 0,
\end{gathered}
$$

shall vanish at more than $m-1$ points of $C$. It is immaterial whether one studies on the one hand the approximation in a closed Jordan region $C$ by harmonic polynomials of a function harmonic interior to the region, continuous in the closed region, or on the other hand the approximation on the boundary of the region of the boundary values of the harmonic function. For the maximum in $C$ of the function in $\left(10^{\prime}\right)$ must occur on the boundary of $C$. Fréchet has proved that the Tchebycheff trigonometric polynomial is unique for approximation to an arbitrary continuous function $f(\theta)$ with period $2 \pi$ on the interval $0 \leqq \theta \leqq 2 \pi$. That is, from our present standpoint (compare \$1), the Tchebycheff harmonic polynomial is unique for approximation to a continuous function on a circumference $C$, or for approximation on and within a circle to a function continuous there, harmonic in the interior. This statement is true, however, only with a restriction. The Tchebycheff polynomial which is a linear combination of the functions $1, r \cos \theta, r \sin \theta, \cdots, r^{n} \cos n \theta, r^{n} \sin n \theta$ is indeed unique, for an arbitrary linear combination $\left(10^{\prime \prime}\right)$ of these functions vanishes on an algebraic curve of degree $n$, and this curve has at most $2 n$ points in common with the circumference $C$. $^{*}$ In fact, this method proves that the corresponding Tchebycheff harmonic polynomial is unique for approximation on an ellipse $C$ to a function continuous on $C$, or for approximation on and within $C$ of a function harmonic interior to $C$, continuous in the corresponding closed region.

The Tchebycheff polynomial, which is a linear combination

* The circumference cannot be a branch of this curve, for the function $\left(10^{\prime \prime}\right)$ cannot vanish everywhere on any (limited) Jordan curve. 
of the functions $1, r \cos \theta, r \sin \theta, \cdots, r^{n-1} \cos (n-1) \theta$, $r^{n-1} \sin (n-1) \theta, r^{n} \cos n \theta$, is not necessarily unique either when $C$ is a circle or an ellipse. Indeed, if $n=1$, the function $\left(10^{\prime \prime}\right)$ is $A_{1}+A_{2} x$, which obviously for suitable choice of $A_{1}$ and $A_{2}$ vanishes at more than a single point of the circle or ellipse $C$.

It would be an interesting investigation, to determine what algebraic curves $C$ have this property, that the Tchebycheff harmonic polynomial for approximation to an arbitrary function continuous on $C$ is unique. This is a subject dealing with the real intersections of plane algebraic curves, in which a single point of intersection counts merely as a single point, in spite of singularities and multiple points (in the usual sense of the term) of either curve.

Whether or not the Tchebycheff harmonic polynomial $t_{n}(x, y)$ of degree $n$ is unique, a Tchebycheff polynomial $t_{n}(x, y)$ of degree $n$ exists, and we can derive certain properties of the sequence $\left\{t_{n}(x, y)\right\}$. In the notation of the previous theorem, we shall prove the following facts.

Let the function $u(x, y)$ be harmonic in the closed interior of $C$, and have at least one singularity on the curve $C_{\rho}$ but no singularity interior to $C_{\rho}$. Then a sequence $\left\{t_{n}(x, y)\right\}$ of Tchebycheff harmonic polynomials for the function $u(x, y)$ considered in the closed interior of $C$ (or what is essentially the same, considered on $C$ itself), whether or not the Tchebycheff polynomial is unique, converges throughout the interior of $C_{\rho}$, and uniformly on any closed point set interior to $C_{\rho}$. The limit of the sequence throughout the interior of $C_{\rho}$ is $u(x, y)$. The sequence can converge uniformly in no region $C_{\rho}$, with $\rho^{\prime}>\rho$.

The proof is immediate. There exists, by the theorem already stated, some sequence $\left\{p_{n}(x, y)\right\}$, where $p_{n}(x, y)$ is of degree $n$, such that we have

$$
\left|u(x, y)-p_{n}(x, y)\right| \leqq \frac{M}{R^{n}}, \quad(x, y) \text { in } C,
$$

provided merely that $R<\rho$. If (10) is valid for the poly- 
nomial $p_{n}(x, y)$, it is also valid if $p_{n}(x, y)$ is replaced by $t_{n}(x, y)$ :

$$
\left|u(x, y)-t_{n}(x, y)\right| \leqq \frac{M}{R^{n}}, \quad(x, y) \text { in } C,
$$

provided still that $R<\rho$. Again by virtue of the theorem stated, the sequence $\left\{t_{n}(x, y)\right\}$ must converge interior to every $C_{R}$ for which $R<\rho$, hence throughout the interior of $C_{\rho}$, uniformly on any closed point set interior to $C_{\rho}$. In particular, if the function $u(x, y)$ has no singularity except at infinity, the sequence converges at every point of the plane, uniformly on any limited closed point set. In the general case, the sequence $\left\{t_{n}(x, y)\right\}$ cannot converge uniformly on any curve $C_{\rho^{\prime}}$ for which $\rho^{\prime}>\rho$, for then it would likewise converge uniformly in a region containing $C_{\rho}$, which has on it a singularity of the function $u(x, y)$.

The theorem just established is the analog of a theorem due to Faber [2, p. 105] for the case of approximation to an analytic function by polynomials in the complex variable. The proofs are, however, different, and the present proof applies without essential change in that other situation, even if $C$ is a Jordan arc or a certain more general point set, instead of a Jordan region.

It will be noticed that the sequence $\left\{t_{n}(x, y)\right\}$ may converge for certain points $(x, y)$ exterior to $C_{\rho}$. Let us suppose for instance that the region $C$ is symmetric on the $x$-axis and that the function $u(x, y)$ satisfies the equation $u(x, y)=-u(x,-y)$. Then a given $t_{n}(x, y)$ may be replaced by another Tchebycheff polynomial $t_{n}^{\prime}(x, y)$ of degree $n$ which satisfies the equation $t_{n}^{\prime}(x, y)=-t_{n}^{\prime}(x,-y)$ and which approximates $u(x, y)$ on $C$ as closely as does $t_{n}(x, y)$. If we have

$$
\left|u(x, y)-t_{n}(x, y)\right| \leqq \epsilon, \quad(x, y) \text { on } C,
$$

we have likewise by symmetry

$$
\left|u(x,-y)-t_{n}(x,-y)\right| \leqq \epsilon, \quad(x, y) \text { on } C,
$$


that is,

$$
\left|u(x, y)+t_{n}(x,-y)\right| \leqq \epsilon, \quad(x, y) \text { on } C
$$

If we set

$$
t_{n}^{\prime}(x, y)=\frac{t_{n}(x, y)-t_{n}(x,-y)}{2}
$$

the harmonic polynomial $t_{n}^{\prime}(x, y)$ obviously satisfies the functional equation considered, and we have the inequality

$$
\left|u(x, y)-t_{n}^{\prime}(x, y)\right| \leqq \epsilon, \quad(x, y) \text { on } C ;
$$

that is to say, the polynomial $t_{n}^{\prime}(x, y)$ is as good an approximation to $u(x, y)$ on $C$ as is $t_{n}(x, y)$. $^{*}$ Interpret $t_{n}^{\prime}(x, y)$ as a linear combination of the functions $r^{n} \cos n \theta, r^{n} \sin n \theta$, hence as a linear combination of the functions $r^{n} \sin n \theta$. The sequence $\left\{t_{n}^{\prime}(x, y)\right\}$ converges for $\theta=0$ for all values of $r$, no matter how small $C$ may be or where the singularities of $u(x, y)$ may lie.

We have thus far restricted our entire discussion of the degree of approximation by harmonic polynomials to the consideration of point sets $C$ which are regions. That is probably the simplest case; the study of the same problems where $C$ is, let us say, a Jordan arc, is more complicated. Complication arises because a harmonic polynomial may vanish identically on $C$, so that convergence on $C$ of a sequence of harmonic polynomials, even so that $(10)$ is satisfied on $C$, does not imply convergence of the sequence for points not on $C$. Let us treat here in detail the simplest pos-

* We have essentially proved here that if the function $u(x, y)$ can be uniformly expanded by harmonic polynomials on a point set $C$ which is symmetric in the $x$-axis and if we have $u(x, y)=-u(x,-y)$, then $u(x, y)$ can likewise be uniformly expanded on $C$ by harmonic polynomials $p_{n}(x, y)$ which satisfy the equation $p_{n}(x, y)=-p_{n}(x,-y)$.

Another theorem of the same general nature easily proved by methods already used elsewhere for functions of a complex variable is that if an arbitrary function $u(x, y)$ can be uniformly expanded by harmonic polynomials on a point set $C$, then $u(x, y)$ is the uniform limit of a sequence of harmonic polynomials each of which is equal to $u(x, y)$ in $n$ arbitrary preassigned points of $C$. 
sible case, namely that $C$ is an interval of the axis of reals; the result is also valid if $C$ is any line segment.

Suppose that $u(x, y)$ is defined on $C$ and that a set of polynomials $\left\{p_{n}(x, y)\right\}$ exists so that (10) holds on $C$ for $R>1$. On $C$ a harmonic polynomial of degree $n$ is a polynomial in $x$ of degree $n$. Inequality (10), valid for a polynomial $p_{n}(x, y)$ in $x$ of degree $n$, implies the convergence of this sequence of polynomials considered as polynomials in the complex variable $x$ throughout the interior of $C_{R}$ (which is defined here as already indicated for a region $C$ ), uniformly on any closed point set interior to $C_{R}$, and hence represents interior to $C_{R}$ an analytic function of the complex variable $x$. This is indeed a theorem due to Bernstein, and in the present case the curve $C_{R}$ is a certain ellipse whose foci are the extremities of the interval $C$. It follows that there exists a function $U(x, y)$, harmonic interior to $C_{R}$, coinciding on $C$ with the given function $u(x, y)$. There are two important differences between this result and the result established for the case that $C$ is a Jordan region. First, we have not shown in the present case, nor is it necessarily true, that the original given sequence of harmonic polynomials $\left\{p_{n}(x, y)\right\}$ converges everywhere interior to $C_{R}$. Second, the function $U(x, y)$ is not uniquely determined by the requirements of being harmonic interior to $C_{R}$ and coinciding on $C$ with the given function $u(x, y)$; if any such function $U(x, y)$ is at hand, we may find another by adding to it an arbitrary function harmonic interior to $C_{R}$ and vanishing on $C$.

Reciprocally, if it is desired to establish (10) when the function $u(x, y)$ is given harmonic on and within $C_{R}$, that can always be done if $C$ is an arbitrary Jordan arc, or indeed a much more general point set. The result follows from the corresponding result for the development of analytic functions in terms of polynomials in the complex variable [compare Walsh, 3].

Before we leave the subject of approximation by harmonic polynomials and turn to harmonic rational functions, we mention another topic which seems not to have been treated 
in the literature and yet which deserves to be investigated, namely, interpolation by means of harmonic polynomials. When does a harmonic polynomial of degree $n$ exist which takes on preassigned values at $2 n+1$ points? When is it unique? What of the asymptotic character of the polynomial as the number of points becomes infinite? What application is there to approximation and expansion, if these points are chosen on the boundary of a region? These questions have been answered in special cases, corresponding to trigonometric interpolation [see Faber, 3, Jackson, 1, de la Vallée-Poussin, 1], and also for the analogous problem of interpolation by polynomials in the complex variable [Fejér, 1]. The former case yields satisfactory results (compare \$1) for interpolation by harmonic polynomials either on a circumference or on and within a circle, but for more general situations the questions seem still to be untouched.*

5. General Approximation by Harmonic Rational Functions. The results thus far established for approximation by harmonic polynomials have precise analogs for approximation of arbitrary harmonic functions by harmonic rational functions; these analogs will now be treated in the same order, and the proofs follow, in the main, the proofs for the simpler case. The results in the present case, however, are mostly new to the literature and must therefore be treated here in some detail. This newness explains the apparent lack of balance between the treatment of the two cases of approximation, by harmonic polynomials and by more general harmonic rational functions.

* These results of Fejér do yield simple theorems immediately. Thus we may make the following statement.

Let $C$ be an arbitrary Jordan curve, and let the function $u(x, y)$ be harmonic on and within $C$. Then there exists a sequence of harmonic polynomials $\left\{p_{n}(x, y)\right\}$ of respective degrees $n$ coinciding with $u(x, y)$ at $n$ points $P_{i}$ of $C$, which converge uniformly on and within $C$ to the function $u(x, y)$. These $n$ points $P_{i}$ can be chosen as points of $C$ which correspond, under conformal mapping of the exterior of $C$ on the exterior of a circle $K$ so that the points at infinity correspond to the vertices of a regular polygon of $n$ sides inscribed in $K$. 
Let the function $u(x, y)$ be harmonic in the closed region $C$ bounded by two analytic Jordan curves $C_{1}$ and $C_{2}$, with $C_{2}$ interior to $C_{1}$ and the origin interior to $C_{2}$. Green's formula

$$
u(x, y)=\frac{1}{2 \pi} \int_{C_{1}+C_{2}}\left[u \frac{\partial \log r}{\partial n}-\log r \frac{\partial u}{\partial n}\right] d s,
$$

where the integrals are to be taken in the positive sense with respect to the region, $n$ being the inner normal, breaks up the function $u(x, y)$ into a function harmonic on and interior to $C_{1}$, plus a function harmonic on and exterior to $C_{2}$, plus a multiple of $\log r$, where $r^{2}=x^{2}+y^{2}$ [see Osgood, 1, pp. 642644 , Walsh, 1, p. 206]. Consequently the function $u(x, y)$ can be approximated in the closed region $C$ as closely as desired by a harmonic polynomial in $(x, y)$ plus a harmonic polynomial in

$$
\left(\frac{x}{x^{2}+y^{2}}, \frac{y}{x^{2}+y^{2}}\right)
$$

plus a multiple of $\log r$. A similar situation obtains if $C$ is bounded not by two Jordan curves but by $n$ Jordan curves, and is not materially altered if these bounding curves are not analytic, nor if $u(x, y)$ is not harmonic in the closed region, but is continuous in the closed region and harmonic in the interior.

We are in a position however to establish a more general* theorem.

Let $C$ be a closed point set which does not contain the point at infinity and which contains no region of infinite connectivity not included in a larger region of finite connectivity belonging to $C$. Let $f(x, y)$ be an arbitrary function continuous on $C$ and

* More general, that is, so far as concerns the point sets and functions considered; actually less specific in one regard, for the older theorem splits up the given function into $2 n-1$ functions, of which $n-1$ are logarithms and left unchanged in the approximation. The other $n$ functions are harmonic in simply-connected regions in whose interiors $C$ lies, and each of these $n$ functions can be uniformly approximated or expanded in terms of harmonic rational functions in the corresponding simply-connected region. 
harmonic in the interior points of $C$. Then if an arbitrary positive $\epsilon$ be given, there exists a harmonic function $\phi(x, y)$, namely, a polynomial in $(x, y)$ and in

$$
\left(\frac{x-x_{i}}{\left(x-x_{i}\right)^{2}+\left(y-y_{i}\right)^{2}}, \frac{y-y_{i}}{\left(x-x_{i}\right)^{2}+\left(y-y_{i}\right)^{2}}\right),
$$

such that we have

$$
\begin{aligned}
& \mid f(x, y)-\phi(x, y)-a_{1} \log r_{1} \\
& \quad-a_{2} \log r_{2}-\cdots-a_{m} \log r_{m} \mid<\epsilon, \quad(x, y) \text { in } C,
\end{aligned}
$$

where the $a_{i}$ are suitable constants and $r_{i}{ }^{2}=\left(x-x_{i}\right)^{2}+\left(y-y_{i}\right)^{2}$. Here the points $\left(x_{i}, y_{i}\right), i=1,2, \cdots$, are exterior to $C$, and can be preassigned, one in each of the regions $R$ into which $C$ separates the plane, although all of such preassigned points do not necessarily appear in (11).

In particular if $C$ has no interior points, an arbitrary function $f(x, y)$ continuous on $C$ can be so approximated.

Let us outline the proof; the method is essentially due to Lebesgue. Let $K$ be a circle which contains $C$. There exists a function $F(x, y)$ continuous on and within $K$ and which coincides with $f(x, y)$ on $C$. There exists a polynomial $p(x, y)$ in $(x, y)$ which is not necessarily harmonic but which throughout $K$ differs from $F(x, y)$ by less than $\epsilon / 3$. Divide the plane into squares and continue subdivision indefinitely, by halving the sides of the squares already constructed, so that we determine a sequence of closed point sets $S_{i}$, each of which consists of a finite number of regions each bounded by a finite number of non-intersecting Jordan curves, of such a nature that each $S_{i}$ contains $C$ in its interior, so that each $S_{i}$ contains its successors, but so that every point exterior to $C$ is exterior to some $S_{i}$. By suitable modification of these point sets $S_{i}$ if necessary, we can make sure that no two of the closed regions composing any particular $S_{i}$ have a point in common. Let $h_{i}(x, y)$ be the function harmonic throughout the interior of $S_{i}$, continuous on $S_{i}$, and coinciding on the boundary of $S_{i}$ with the function $p(x, y)$. 
Then we have [Walsh, 1, p. 199] $\lim _{i \rightarrow \infty} h_{i}(x, y)=p(x, y)$ uniformly on the boundary points of $C$. That is, in particular we can choose $k$ so that $\left|h_{k}(x, y)-p(x, y)\right|<\epsilon / 3$ uniformly on the boundary of $C$. But on $C$ the function $h_{k}(x, y)$ can be uniformly approximated by a function of the sort considered in the theorem:

$$
\begin{aligned}
\mid h_{k}(x, y)-\phi(x, y) & -a_{1} \log r_{1} \\
& -\cdots-a_{m} \log r_{m} \mid<\frac{\epsilon}{3},(x, y) \text { on } C,
\end{aligned}
$$

and the points $\left(x_{i}, y_{i}\right)$ lie exterior to $C$. In fact, if the points $\left(x_{i}, y_{i}\right)$ are not preassigned, we can [loc. cit., p. 208] satisfy (12) on the entire point set $S_{k}$. If the points $\left(x_{i}, y_{i}\right)$ are preassigned, we can make the approximation (12) not on the point set $S_{k}$ but on a point set $S_{k}^{\prime}$ of the same connectivity as $S_{k}$, bounded by a finite number of non-intersecting Jordan curves, which is contained in $S_{k}$, which contains $C$, but which contains precisely those of the preassigned points $\left(x_{i}, y_{i}\right)$ which lie in regions $R$ lying entirely in $S_{k}$. In this new approximation (12), which holds in $S_{k}^{\prime}$ and hence in $C$, we use only the preassigned points $\left(x_{i}, y_{i}\right)$.

Combination of the inequalities obtained yields (11) uniformly for all points on the boundary of $C$. But all the functions in the left-hand member of (11) are harmonic in the interior points of $C$, continuous on the corresponding closed point set, and such a function has no maximum or minimum in an interior point. Hence inequality (11) holds for all points of $C$, and the theorem is established.

We mention explicitly that it is not true that an arbitrary function continuous in a limited closed region and harmonic interior to the region can be uniformly approximated in that region as closely as desired by a harmonic rational function, either with or without logarithmic terms as considered in (11). Consider for example the region $C$ formed from the circle $x^{2}+y^{2}<1$ by cutting out the line segment $y=0$, $-1 / 2 \leqq x \leqq 1 / 2$; let $f(x, y)$ be the function harmonic interior 
to this region, continuous in the closed region, zero on the circumference, and unity on the line segment. The function $f(x, y)$ cannot be uniformly approximated in the closed region. For the approximating functions have only isolated singularities [Osgood 1, p. 680], are continuous without exception in the closed region (else are not uniformly bounded), hence are harmonic in the closed region. These approximating functions can be uniformly approximated as closely as desired in the closed region $C$ by harmonic polynomials in $(x, y)$, so $f(x, y)$ can also be uniformly approximated in $C$ as closely as desired by a harmonic polynomial in $(x, y)$. But if such a polynomial differs from $f(x, y)$ by less than $\epsilon$ for points on $x^{2}+y^{2}=1$, that polynomial differs from zero by less than $\epsilon$ for all points $x^{2}+y^{2}<1$, which is a contradiction for $x=y=0$ if $\epsilon<1 / 2$.

The general theorem we have proved, culminating in inequality (11), is obviously not an exhaustive discussion of its subject-matter. If $C$ is a closed region of infinite connectivity, is it true that an arbitrary function $f(x, y)$ harmonic interior to $C$ and continuous in the closed region, can in the closed region be uniformly approximated as closely as desired, as in (11)? Is our general theorem true without any restriction as to regions of infinite connectivity? Is it true that if $C$ is an arbitrary closed point set without interior points, then an arbitrary function $f(x, y)$ continuous on $C$ can be uniformly approximated on $C$ as closely as desired by a rational harmonic function without logarithmic terms?

Even though we are not in a position to answer this last question, there are specific closed point sets $C$ on which an arbitrary continuous function can certainly be approximated as closely as desired by a harmonic rational function. In fact, an arbitrary closed point set $C$ which consists of a finite number of Jordan arcs which do not divide the plane into an infinite number of regions has this property. More generally, [compare Walsh, 5] if $C$ is such a point set that an arbitrary function continuous on $C$ can be approximated on $C$ as closely as desired by a rational function of the complex variable, then 
$C$ also has the property considered. For let $f(x, y)$ be the given (real) function; a rational function $p(z)$ of $z$ exists so that we have $|f(x, y)-p(z)|<\epsilon,(x, y)$ on $C$, where $\epsilon$ is preassigned. Hence we have also $|f(x, y)-r(x, y)|<\epsilon,(x, y)$ on $C$, where $r(x, y)$ is a rational harmonic function of $(x, y)$, the real part of $f(z)$.

We do not attempt to approximate the most general function harmonic in a multiply-connected region $C$ by harmonic rational functions without the use of logarithmic terms, for that is impossible, as we shall now prove, if the closed region cannot be considered as a closed simply-connected region.* We choose $C$ limited, so there exists an analytic Jordan curve $J$ interior to $C$, whose interior contains points not belonging to the closed region $C$; let us assume the origin to be such a point, so that the function $\log r$, where $r^{2}=x^{2}+y^{2}$, is harmonic in the closed region $C$. Assume the approximation possible, so that $\log r$ can be expanded in $C$ in a uniformly convergent series of harmonic rational functions. These rational functions have only isolated singularities and are continuous in $C$, hence harmonic throughout the interior of $C$. Differentiate this series term by term in the direction of the normal to $J$, and integrate the resulting series over $J$ term by term [Osgood 1, pp. 652-653]. For each term of the series, the result of this process is zero [Osgood 1, p. 680], but for the function $\log r$ the result is $2 \pi$. We thus have the contradiction $2 \pi=0$, and the statement is established.

The entire discussion we have given enables us to answer the question as to whether a function given on an arbitrary closed point set $C$ can be uniformly approximated on that point set by harmonic rational functions plus logarithmic terms, with singularities in assigned points not belonging to $C$, provided that no region of infinite connectivity is involved. The facts and proofs are so similar to the corresponding facts and proofs for the case of approximation by harmonic polynomials [Walsh, 1] that they are omitted.

* Already proved [Walsh 1, p. 206] in a special case. 
It is not our purpose to study non-uniform expansion of harmonic functions, but one result is now interesting and yet so obvious that we mention it here; it is still an open problem to determine in general what functions can be expanded not necessarily uniformly in terms of harmonic rational functions either with or without logarithmic terms.

Let $C_{0}$ be an arbitrary open point set, which may be empty. Let $C_{1}, C_{2}, \cdots$ be closed point sets (any or all of which may be empty) mutually exclusive and having no common point with $C_{0}$, and on each of the point sets $C_{0}, C_{1}, \cdots$ let the function $u(x, y)$ be expansible by harmonic rational functions plus logarithmic terms. Then the function $u(x, y)$ can be expanded on $C_{0}+C_{1}$ $+C_{2}+\cdots$ in a series of rational harmonic functions plus logarithmic terms. The series converges uniformly on each of the point sets $C_{1}, C_{2}, \cdots$ on which $u(x, y)$ is uniformly expansible, and if $u(x, y)$ is harmonic on $C_{0}$, except possibly for logarithmic singularities or singularities corresponding to rational harmonic functions, then the series converges uniformly on every closed point set contained in $C_{0}$.

We use $\psi(x, y)$ generically to denote a rational harmonic function with logarithmic terms, such as occurs in (11). Let $S_{1}, S_{2}, \cdots$ be closed point sets, each consisting of a finite number of mutually exclusive regions, each bounded by a finite number of non-intersecting Jordan curves, and such that $S_{k}$ lies interior to $C_{0}$, that $S_{k}$ lies interior to $S_{k+1}$, and that every point of $C_{0}$ lies in some $S_{k}$. Let us suppose

$$
u(x, y)=\lim _{n \rightarrow \infty} \psi_{k n}(x, y), \quad\left[(x, y) \text { on } C_{k}, k=0,1,2, \cdots\right],
$$

where this limit is approached uniformly if $u(x, y)$ is uniformly expansible on $C_{k}, k \geqq 1$, and uniformly on every closed point set in $C_{0}$ for $k=0$ if that is possible. Then we can determine a closed point set $B_{11}$, consisting of a finite number of mutually exclusive closed regions each bounded by a finite number of non-intersecting Jordan curves, which contains $C_{1}$ but has no point in common with $S_{1}$. We can also determine $\psi_{1}(x, y)$ (see the lemma below) such that 


$$
\begin{aligned}
& \left|\psi_{1}(x, y)-\psi_{02}(x, y)\right|<\frac{1}{2}, \\
& \left|\psi_{1}(x, y)-\psi_{11}(x, y)\right|<\frac{1}{2},
\end{aligned}
$$$$
(x, y) \text { on } S_{1} \text {, }
$$$$
(x, y) \text { on } B_{11} \text {. }
$$

Let $B_{12}$ be a closed point set of the topological simplicity of $B_{11}$, which contains $C_{1}$ but has no point in common with $S_{2}$ or $C_{2}$. Let $B_{21}$ be a closed point set likewise of the topological simplicity of $B_{11}$, which contains $C_{2}$ but has no point in common with $S_{2}$ or $B_{12}$. Choose $\psi_{2}(x, y)$ so that we have

$$
\begin{array}{ll}
\left|\psi_{2}(x, y)-\psi_{03}(x, y)\right|<\frac{1}{3}, & (x, y) \text { on } S_{2}, \\
\left|\psi_{2}(x, y)-\psi_{12}(x, y)\right|<\frac{1}{3}, & (x, y) \text { on } B_{12}, \\
\left|\psi_{2}(x, y)-\psi_{21}(x, y)\right|<\frac{1}{3}, & (x, y) \text { on } B_{21} .
\end{array}
$$

We continue this process; in general $B_{1 n}$ shall contain $C_{1}$ but shall have no point in common with $S_{n}$ or $C_{2}, C_{3}, \cdots$, $C_{n}$. The point set $B_{2, n-1}$ shall contain $C_{2}$ but shall have no point in common with $C_{3}, \cdots, C_{n}, S_{n}$ or $B_{1 n}$. The point set $B_{n 1}$ shall contain $C_{n}$ but shall have no point in common with $S_{n}$ or $B_{1 n}, B_{2, n-1}, \cdots, B_{n-1,2}$. The function $\psi_{n}(x, y)$ is then to be chosen so that we have

$$
\begin{aligned}
& \left|\psi_{n}(x, y)-\psi_{0, n+1}(x, y)\right|<\frac{1}{n+1}, \quad(x, y) \text { on } S_{n}, \\
& \left|\psi_{n}(x, y)-\psi_{1 n}(x, y)\right|<\frac{1}{n+1}, \quad(x, y) \text { on } B_{1 n}, \\
& \left|\psi_{n}(x, y)-\psi_{n 1}(x, y)\right|<\frac{1}{n+1}, \quad(x, y) \text { on } B_{n 1},
\end{aligned}
$$

and the sequence $\left\{\psi_{n}(x, y)\right\}$ has the property required in the theorem. Uniform convergence of the sequence $\left\{\psi_{n}(x, y)\right\}$ on a closed point set belonging to $C_{0}+C_{1}+\cdots$ but not necessarily a $C_{k}$ depends merely on the uniform convergence of the corresponding sequence (or sequences) $\left\{\psi_{k n}(x, y)\right\}$ on that point set. The function $u(x, y)$, if harmonic on $C_{0}$ except possibly for logarithmic singularities or singularities corresponding to rational harmonic functions, can be chosen arbitrarily on $C_{0}$, and the sequence $\left\{\psi_{n}(x, y)\right\}$ converges to the 
value $u(x, y)$, uniformly on any closed point set contained in $C_{0}$.

If none of the point sets $C_{0}, C_{1}, C_{2}, \cdots$ separates any point of another of those point sets from the point at infinity the theorem is true if the words harmonic rational functions plus logarithmic terms are replaced by harmonic polynomials. For uniform convergence on any closed point set contained in $C_{0}$, however, we require that $u(x, y)$ should be harmonic in $C_{0}$ and that $C_{0}$ should be composed of mutually exclusive simply-connected regions. In the discussion just given we have had occasion to apply the following lemma.

Lemma. If $S_{1}$ and $S_{2}$ are mutually exclusive closed point sets each consisting of a finite number of mutually exclusive regions each bounded by a finite number of non-intersecting Jordan curves, and if the functions $\psi_{1}(x, y)$ and $\psi_{2}(x, y)$ are rational harmonic functions with logarithmic terms, then if $\epsilon>0$ be given there exists a rational harmonic function $\psi(x, y)$ with logarithmic terms so that we have

$$
\begin{array}{ll}
\left|\psi(x, y)-\psi_{1}(x, y)\right|<\epsilon, & (x, y) \text { in } S_{1}, \\
\left|\psi(x, y)-\psi_{2}(x, y)\right|<\epsilon, & (x, y) \text { in } S_{2} .
\end{array}
$$

For simplicity in the proof we assume that both $S_{1}$ and $S_{2}$ are limited. We write $\psi_{1}(x, y)=\psi_{1}^{\prime}(x, y)+\psi_{1}^{\prime \prime}(x, y)$, where $\psi_{1}^{\prime}(x, y)$ has no singularities in $S_{1}$ and $\psi_{1}^{\prime \prime}(x, y)$ has none in $S_{2}$. Similarly set $\psi_{2}(x, y)=\psi_{2}^{\prime}(x, y)+\psi_{2}^{\prime \prime}(x, y)$, where $\psi_{2}^{\prime}(x, y)$ has no singularities in $S_{2}$, and $\psi_{2}^{\prime \prime}(x, y)$ none in $S_{1}$; this splitting up is possible in the present case, but a slight modification may be necessary, due to the presence of logarithmic terms, if the point at infinity belongs to $S_{1}$ or $S_{2}$. It is possible [Walsh, 1, p. 208] to determine $\psi^{\prime}(x, y)$ so that the inequalities

$$
\begin{array}{ll}
\left|\psi^{\prime}(x, y)-\psi_{1}^{\prime}(x, y)+\psi_{2}^{\prime \prime}(x, y)\right|<\epsilon, & (x, y) \text { in } S_{1} \\
\left|\psi^{\prime}(x, y)-\psi_{2}^{\prime}(x, y)+\psi_{1}^{\prime \prime}(x, y)\right|<\epsilon, & (x, y) \text { in } S_{2}
\end{array}
$$

are satisfied. These inequalities, if we set

$$
\psi(x, y)=\psi^{\prime}(x, y)+\psi_{1}^{\prime \prime}(x, y)+\psi_{2}^{\prime \prime}(x, y)
$$


are the inequalities it is desired to establish.

We mention explicitly the general question of the possibility of the expansion of given functions in terms of harmonic polynomials or in terms of harmonic rational functions when the restriction of uniformity of convergence is not made. This question seems not to be completely answered in the literature, although Lavrentieff [1] has recently announced some results without proof, and Hartogs and Rosenthal [1] have published an important paper on the corresponding subject for expansion in terms of polynomials in the complex variable. It is worth noting that Osgood's classical theorem [2] in this corresponding subject has the following analog in the present one.

If there converges in a region $R$ a sequence of functions $\left\{u_{n}(x, y)\right\}$ harmonic in $R$, then this sequence converges uniformly in some sub-region of $R$.

From this theorem follows directly Osgood's better known result, that if there converges in a region $R$ a sequence of functions $\left\{f_{n}(z)\right\}$ analytic in that region, then this sequence converges uniformly in some sub-region of $R$. For since the sequence $\left\{f_{n}(z)\right\}$ converges, the sequence $\left\{u_{n}(x, y)\right\}$ of the real parts of these functions converges in $R$, hence uniformly in some sub-region $R^{\prime}$ of $R$. Convergence of the sequence $\left\{i v_{n}(x, y)\right\}$ of the pure imaginary parts of the functions $f_{n}(z)$ at a single point of $R^{\prime}$ is now sufficient to ensure convergence of the sequence $\left\{i v_{n}\right\}$ and hence of the sequence $\left\{f_{n}(z)\right\}$ uniformly in any simply-connected closed proper sub-region of $R^{\prime}$.

Let us indicate briefly the proof of the theorem for harmonic functions. We prove first, after Montel [1, p. 109], but this is only a modification of Osgood's proof, that the sequence $\left\{u_{n}(x, y)\right\}$ is uniformly bounded in some sub-region of $R$. Otherwise we should have some $\left|u_{n_{1}}(x, y)\right|>1$ at some point of $R$, hence in some sub-region $R_{1}$ of $R$. If the sequence $\left\{u_{n}(x, y)\right\}$ is not uniformly bounded in $R_{1}$, we must have $\left|u_{n_{2}}(x, y)\right|>2$ at some point of $R_{1}$, and therefore in some sub-region $R_{2}$ of $R_{1}$. Proceeding in this way we arrive at 
a sequence $\left\{u_{n_{k}}(x, y)\right\}$ greater in absolute value than $\{k\}$ in $R_{k}$. There is at least one point common to all the $R_{k}$, and at this point the original sequence cannot converge.

From the boundedness of the original sequence in some sub-region $R^{\prime}$ it follows [compare Osgood, 2] that the first partial derivatives of these functions are uniformly bounded in an arbitrary closed sub-region of $R^{\prime}$, hence that the functions $\left\{u_{n}(x, y)\right\}$ are equicontinuous in this sub-region and therefore converge uniformly there.

We shall not go into great detail on the subject of rational harmonicfunctions belonging to a region, for the discussion can be made to depend upon the discussion for harmonic polynomials belonging to a region. Let a region $C$ be bounded by the Jordan curves $C_{0}, C_{1}, \cdots, C_{k}$, of which no two have a common point, and so that the curves $C_{1}, C_{2}, \cdots, C_{k}$ lie interior to $C_{0}$. Let $u(x, y)$ be an arbitrary function harmonic interior to $C$ and continuous in the closed region. As has already been suggested, Green's formula applied to $u(x, y)$ not for the region $C$ but for a neighboring region $C^{\prime}$ of the same connectivity interior to $C$ splits up the function $u(x, y)$ into $k$ logarithmic terms with singularities at infinity and at points interior to $C_{1}, C_{2}, \cdots, C_{k}$ respectively, which may be preassigned, plus $k$ functions harmonic exterior respectively to $C_{1}, C_{2}, \cdots, C_{k}$, including the point at infinity, and continuous in the corresponding closed regions, plus a function harmonic interior to $C_{0}$ and continuous in the corresponding closed region. If the curves $C_{0}, C_{1}, \cdots, C_{k}$ are suitably restricted, the regions interior to $C_{0}$ and exterior to $C_{i}(i=1,2, \cdots, k)$ respectively have associated with them (compare §3) sets of rational functions, in fact polynomials in $(x, y)$ and in

$$
\left(\frac{x-x_{i}}{\left(x-x_{i}\right)^{2}+\left(y-y_{i}\right)^{2}}, \frac{y-y_{i}}{\left(x-x_{i}\right)^{2}+\left(y-y_{i}\right)^{2}}\right)
$$

respectively, and the last mentioned $k+1$ functions can be respectively expanded in terms of these functions.

It would be an interesting problem, however, to construct 
for the region $C$ a single set of normal orthogonal harmonic rational functions (with also $k$ logarithmic functions) in terms of which an arbitrary harmonic function could be expanded. Here orthogonal may mean (1) with respect to area (that is, surface integral over $C$ ) or (2) with respect to length (that is, line integral over $C_{0}, C_{1}, \cdots, C_{k}$ ). Such a set of functions can easily be constructed by the process of orthogonalization, starting with the logarithmic and rational functions we have been using. If interpretation (2) is used, it is true that if the curves $C_{0}, C_{1}, \cdots, C_{k}$ bounding the region $C$ are analytic, and if the function $u(x, y)$ is harmonic interior to $C$, continuous in the corresponding closed region, then the formal expansion of $u(x, y)$ in terms of these normal orthogonal functions belonging to $C$ converges to the value $u(x, y)$ throughout the interior of $C$, uniformly on any closed point set interior to $C$. This follows from the reasoning as given by Merriman [1]; but questions of asymptotic character of the normal orthogonal functions and of uniform convergence of the formal expansion in the closed region $C$, are there left unanswered under the present general hypothesis on the region $C$.

6. Degree of Approximation by Rational Harmonic Functions. Our results on the degree of approximation to a harmonic function by rational harmonic functions are to be obtained with the help of the corresponding results on the degree of approximation to an analytic function by rational functions of the complex variable, so as a preliminary study we need to consider the relation between the degree of a rational harmonic function and the degree of the corresponding analytic function. In this discussion we use the word degree to indicate degree in the restricted sense.*

If $u(x, y)$ is a harmonic polynomal of degree $n$, then its conjugate function $v(x, y)$ is likewise a harmonic polynomial of degree $n$ and hence the function $f(z)=u(x, y)+i v(x, y)$ is a

* Compare $\$ 4$. The degree of a rational function is the greater of the degrees of numerator and denominator, or the common degree if the two are of the same degree. 
polynomial in $z$ of degree $n$. In fact, we may define $v(x, y)$ by

$$
v(x, y)=\int_{(0,0)}^{(x, y)}\left(-\frac{\partial u}{\partial y} d x+\frac{\partial u}{\partial x} d y\right)+C,
$$

where $C$ is an arbitrary constant. The function $f(z)=u(x, y)$ $+i v(x, y)$ can have no singular points other than those of $u(x, y)$, hence is an entire function. In the Taylor's development of $f(z)$ about the origin, the coefficients of powers of $z$ higher than the $n$th vanish; otherwise $u(x, y)$ is not a polynomial of degree $n$.

The situation is somewhat more complicated if $u(x, y)$ is a harmonic rational function not a polynomial. Osgood [1, p. 680] has shown that if $u(x, y)$ is harmonic and rational, then $f(z)=u(x, y)+i v(x, y)$, where $v(x, y)$ is defined by the equation just given, is a rational function of $z$. If $f(z)$ is given, with no factor containing $z$ common to numerator and denominator,

$$
f(z)=\frac{a_{0} z^{m}+a_{1} z^{m-1}+\cdots+a_{m}}{b_{0} z^{n}+b_{1} z^{n-1}+\cdots+b_{n}}, \quad a_{0} b_{0} \neq 0
$$

the real part is found by multiplying numerator and denominator by the conjugate complex quantity of the original denominator,

$$
f(z)=\frac{a_{0} \bar{b}_{0} z^{m} \bar{z}^{n}+\cdots+a_{m} \bar{b}_{n}}{b_{0} \bar{b}_{0} z^{n} \bar{z}^{n}+\cdots+b_{n} \bar{b}_{n}}
$$

and then separating into real and pure imaginary parts. The function $u(x, y)$ is then a rational function whose numerator is of total degree $m+n$ or less, whose denominator is of degree $2 n$, and with no factor common to numerator and denominator; the actual degree of $u(x, y)$ is $m+n$ or less, or $2 n$ according as $m \geqq n$ or $m<n$. Reciprocally, let $u(x, y)$ be given in its lowest terms; denote the degrees of numerator and denominator by $p$ and $2 q$ respectively, and those of the numerator and denominator of the corresponding analytic function $f(z)$, which is determined only to within an additive constant, by $m$ and $n$. A necessary and sufficient condition 
that $u(x, y)$ be harmonic at infinity is that $f(z)$ be analytic at infinity, that is, $m \leqq n$; in this case we have $n=q, m \geqq p-q$; the actual degree of $u(x, y)$ is $2 n$ and that of $f(z)$ is $n$. A necessary and sufficient condition that $u(x, y)$ be singular at infinity is that $f(z)$ be singular at infinity, that is, $m>n$. Here we may write $f(z)$ as a rational function whose numerator is of degree $n-1$ or less and denominator of degree $n$ plus a polynomial of degree $m-n$. Then $u(x, y)$ is a rational function whose numerator is of degree $2 n-1$ or less and denominator of degree $2 n$ plus a polynomial of degree $m-n$, or a rational fraction whose numerator is of degree $p=m+n$ and denominator of degree $2 q=2 n$; the actual degree of $u(x, y)$ is $m+n$ and that of $f(z)$ is $m$.

We can prove the following result concerning approximation by harmonic rational functions; henceforth we use the word degree in its inclusive sense.

Let $S$ be an arbitrary closed Jordan region of the $(x, y)$-plane. If there exist rational harmonic functions $r_{n}(x, y)$, real parts of rational functions of $z=x+i y$ of respective degrees $n^{*}$ such that we have

$$
\left|u(x, y)-r_{n}(x, y)\right| \leqq \frac{M}{R^{n}}, \quad R>1,
$$

for all points $(x, y)$ of $S$ and for all sufficiently large $n$, and if the singular points of the functions $r_{n}(x, y)-r_{n-1}(x, y)$ have no limit point on $S$, then $u(x, y)$ is the real part of a function $f(z)$ meromorphic on $S$. If in addition the functions $r_{n}(x, y)$ have no singularities on $S$, then $u(x, y)$ is harmonic on $S$.

Let $w=\Phi(z)$ denote a function which maps the complement of $S$ onto the exterior of the unit circle in the w-plane, so that the points at infinity correspond to each other. Let $S_{R}$ denote the Jordan curve $|\Phi(z)|=R$, where $R>1$. If the singularities of the functions $r_{n}(x, y)-r_{n-1}(x, y)$ have no limit point interior to

* The rational function of $z$ is not determined uniquely by $r_{n}(x, y)$, but merely to within an additive constant. Nevertheless the degree, and nature and location of the singularities of this rational function are determined by $r_{n}(x, y)$ uniquely. 
$S_{\rho}$, then the sequence $\left\{r_{n}(x, y)\right\}$ converges interior to $S_{\nu}$, where $\nu=\left(1+\rho R^{1 / 2}\right) /\left(\rho+R^{1 / 2}\right)$, and the convergence is uniform on any closed point set interior to $S_{\nu}$. Hence $f(z)$ is meromorphic interior to $S_{\nu}$ and if $r_{n}(x, y)$ has no singularities interior to $S_{\nu}$, the function $u(x, y)$ is harmonic interior to $S_{\nu}$.

If the function $r_{n}(x, y)-r_{n-1}(x, y)$ is the real part of a function which has at most $n$ poles, for $n$ sufficiently large, we may set $\nu=(1+\rho R) /(\rho+R)$, and in particular if the only singularities of $r_{n}(x, y)$ lie at infinity, we may set $\rho=\infty, \nu=R$, so that the sequence $\left\{r_{n}(x, y)\right\}$ converges interior to $S_{R}$.

The proof follows the proof of the corresponding theorem [Walsh 3, Theorem I] for approximation by means of polynomials. If the functions $f(z)$ and $t_{n}(z)$, of which $u(x, y)$ and $r_{n}(x, y)$ are respectively the real parts, are properly chosen, and if $S^{\prime}$ is an arbitrary closed region interior to $S$, then the inequality $\left|f(z)-t_{n}(z)\right| \leqq M^{\prime} / R^{n}$ is valid for $z$ in $S^{\prime}$. If the region $S^{\prime}$ is a region bounded by a Jordan curve uniformly near the boundary of $S$, the function $w=\Phi^{\prime}(z)$ which maps the complement of $S^{\prime}$ onto the exterior of the unit circle in the w-plane differs little from the function $w=\Phi(z)$, and the curve $S_{R}{ }^{\prime}:\left|\Phi^{\prime}(z)\right|=R$ lies uniformly near the curve $S_{R}:|\Phi(z)|=R$. The theorem follows by virtue of the corresponding theorem for approximation to analytic functions by means of rational functions [Walsh 4 , Theorem IV].

It is a positive simplification here not to mention the degree of $r_{n}(x, y)$ except in connection with $t_{n}(z)$, for such widely differing functions as

$$
x, \frac{x}{x^{2}+y^{2}}, \frac{3 x^{2}+3 y^{2}+x}{x^{2}+y^{2}}
$$

belong in the same category.

We turn now to the study of the approximation of functions by rational harmonic functions in multiply connected regions. Here logarithmic terms are essential for the approximation of the most general harmonic functions, as we have seen, so we prove the following theorem. 
Let $C$ be a closed region bounded by Jordan curves $C_{0}, C_{1}$, $\cdots, C_{k}$, such that no two of these curves have a common point and so that $C_{1}, C_{2}, \cdots, C_{k}$ lie interior to $C_{0}$. If the sequence

$$
\begin{aligned}
& r_{n}(x, y)+A_{n 1} \log \left[\left(x-x_{n 1}\right)^{2}+\left(y-y_{n 1}\right)^{2}\right] \\
& \quad+A_{n 2} \log \left[\left(x-x_{n 2}\right)^{2}+\left(y-y_{n 2}\right)^{2}\right] \\
& +\cdots+A_{n k} \log \left[\left(x-x_{n k}\right)^{2}+\left(y-y_{n k}\right)^{2}\right] \\
& \quad(n=1,2, \cdots)
\end{aligned}
$$

converges uniformly in $C$, where $r_{n}(x, y)$ is a harmonic rational function of $(x, y)$ with no singularities interior to $C$, and where the point $\left(x_{n i}, y_{n i}\right)$ lies in or on $C_{i}$ and approaches a limit $\left(x_{i}, y_{i}\right)$ as $n$ becomes infinite, ${ }^{*}$ then $\lim _{n \rightarrow \infty} A_{n i}$ exists, $i=1,2, \cdots$, $k$, and each of the sequences

$r_{n}(x, y), A_{n i} \log \left[\left(x-x_{n i}\right)^{2}+\left(y-y_{n i}\right)^{2}\right],(i=1,2, \cdots, k)$, converges uniformly in any closed region interior to $C$; if the functions in (14) have no singularity in $C$ and no limit point of singularities in $C$, this convergence is uniform in the closed region $C$.

The proof is simple; choose an analytic Jordan curve $C_{i}^{\prime}$ interior to $C$ and enclosing $C_{i}$ but none of the curves $C_{1}$, $C_{2}, \cdots, C_{i-1}, C_{i+1}, \cdots, C_{k}$ in its interior. The sequence of the derivatives of (13) in the direction of the normal $\nu$ to $C_{i}^{\prime}$ converges uniformly on $C_{i}^{\prime}$, and can be integrated on $C_{i}^{\prime}$ term by term [Osgood 1, pp. 652-653, Walsh 1, p. 206]. It follows from the theorem of Osgood already quoted that

$$
\int_{C_{i^{\prime}}} \frac{\partial r_{n}(x, y)}{\partial \nu} d s=0 ;
$$

for we have

$$
\int_{C_{i^{\prime}}} \frac{\partial r_{n}(x, y)}{\partial \nu} d s=\int_{C_{i^{\prime}}} \frac{\partial s_{n}(x, y)}{\partial s} d s=0,
$$

* It is of course sufficient if the point $\left(x_{i}, y_{i}\right)$ lies on or within $C_{i}$ without the assumption that $\left(x_{n i}, y_{n i}\right)$ lies interior to or on $C_{i}$; we integrate (as below) over $C_{i}^{\prime}$ when $n$ is chosen so large that all points $\left(x_{n i}, y_{n i}\right)$ but no points $\left(x_{n i}, y_{n j}\right), i \neq j$, lie interior to $C_{i}^{\prime}$. 
where $s_{n}(x, y)$ is a function conjugate to $r_{n}(x, y)$. The total result of the integration of the $n$th term of the sequence (13) is then precisely $4 \pi A_{n i}$, so $\lim _{n \rightarrow \infty} A_{n i}=A_{i}$ exists. It follows that

$$
\begin{aligned}
\lim _{n \rightarrow \infty} A_{n i} \log \left[\left(x-x_{n i}\right)^{2}+(\right. & \left.\left.y-y_{n i}\right)^{2}\right] \\
& =A_{i} \log \left[\left(x-x_{i}\right)^{2}+\left(y-y_{i}\right)^{2}\right],
\end{aligned}
$$

uniformly in any closed region interior to $C$, and hence it follows that the sequence $\left\{r_{n}(x, y)\right\}$ converges uniformly as asserted.

In the next theorem we shall not trouble to consider sequences so general as (13).

Let $C$ be a closed region bounded by Jordan curves $C_{0}, C_{1}$, $\cdots, C_{k}$, such that no two of these curves have a common point and such that $C_{1}, C_{2}, \cdots, C_{k}$ lie interior to $C_{0} . A$ necessary and sufficient condition that the function $u(x, y)$ defined in $C$ should be harmonic in (the closed region) $C$, is that there should exist rational harmonic functions $r_{n}(x, y)$, real parts of rational functions of $z$ of degrees $(k+1) n, n=0,1,2, \cdots$, so that we have

$$
\begin{aligned}
& \mid u(x, y)-\sum_{i=1}^{k} A_{i n} \log \left[\left(x-x_{i}\right)^{2}\right. \\
& \left.\quad+\left(y-y_{i}\right)^{2}\right]-r_{n}(x, y) \mid \leqq \frac{M}{R^{n}}, \quad R>1,
\end{aligned}
$$

for all points $(x, y)$ of $C$; here the $A_{\text {in }}$ are constants and the points $\left(x_{i}, y_{i}\right)$ are supposed to lie interior to $C_{i}$ respectively. It follows that $\lim _{n \rightarrow \infty} A_{\text {in }}=A_{i}$ exists if (15) is satisfied.

The sufficiency of this condition follows, as in the proof already given, from Osgood's theorem and from the corresponding result for approximation of analytic functions of the complex variable [Walsh 4 , Theorem V]. It is found by the integration that

$$
\left|A_{\text {in }}-A_{i}\right| \leqq M^{\prime} / R_{n}
$$

hence (15) holds if $A_{i n}$ is replaced by $A_{i}$, provided that $M$ is replaced by a suitable $M^{\prime \prime}$. The necessity of the condition is likewise easy to establish. The points $\left(x_{i}, y_{i}\right)$ may be chosen arbitrarily interior to the curves $C_{i}$ respectively. Then [com- 
pare Osgood 1, pp. 642-644; Walsh 1, p. 206] in $C$ the function $u(x, y)$ may be expressed as the sum of the $k$ logarithmic terms which appear in (15) plus $k$ functions harmonic respectively on and exterior to $C_{i}$, and at infinity, for $i=1,2, \cdots, k$, plus a function harmonic in the closed interior to $C_{0}$. The results of $\$ 4$ can now be applied, and yield the desired rational functions $r_{n}(x, y)$.

This theorem can be extended by considering (1) variable logarithmic terms, (2) more explicit regions or point sets for the location of the singularities of the functions $r_{n}(x, y),(3)$ more explicit regions (that is, regions containing $C$ in their interiors) for the harmonic character of $u(x, y)$ and for the convergence of the sequence $\left\{r_{n}(x, y)\right\},(4)$ more general boundaries for regions than Jordan curves. There is comparatively little difficulty involved in making any of these generalizations however, and the essential reasoning involved already appears in the literature (either here or elsewhere), so these generalizations are left to the reader.

It is also possible to study sequences of harmonic rational functions which satisfy such a relation as (15) not in a region but on a Jordan arc or curve. Here the situation is not so simple, as we have indicated in the analogous situation in $\$ 4$. If (15) holds merely on the unit circle $C$, for instance, the sequence of harmonic rational functions need not converge elsewhere; for the function $A_{n} \log r$ vanishes on $C$ and a change in $A_{n}$ does not alter (15), yet may alter the convergence off of $C$ of the sequence in (15). The rational harmonic functions are not uniquely determined, moreover, by their values on $C$; the two functions $r^{n} \cos n \theta$ and $r^{-n} \cos n \theta$ are equal on $C$. Knowledge even of the location of the singularities of a harmonic rational function (such as $a r^{n} \cos n \theta$ $\left.+b r^{-n} \cos n \theta\right)$ and of the value on $C$ does not determine the rational function uniquely. Nor is a function $u(x, y)$ harmonic in a region containing $C$ in its interior determined by its values on $C$.

If (15) holds on the unit circle $C$ for rational functions $\left\{r_{n}(x, y)\right\}$ with singularities only at the origin and at infinity 
and where $\left(x_{i}, y_{i}\right), i=1$, is the origin, there exists a function $U(x, y)$ harmonic on $C$, in fact harmonic for $r<R$, and coinciding on $C$ with the values $u(x, y)$. Indeed, the logarithmic term of (15) vanishes on $C$ and hence may be entirely omitted. The rational function $r_{n}(x, y)$ is a polynomial in $r^{-n} \cos n \theta, r^{-n} \sin n \theta, \cdots, 1, \cos \theta, \sin \theta, \cdots, r^{n} \cos n \theta, r^{n} \sin n \theta$, but the negative powers of $r$ may be changed into positive powers without altering $r_{n}(x, y)$ or (15) on $C$, so we are dealing with a sequence of harmonic polynomials, converging like a geometric series on $C$. This situation has already been treated in $\$ 4$, and the existence of the required function $U(x, y)$ is established there.

7. Expansions in Three Dimensions. The theory of the expansion of harmonic functions in three dimensions is not nearly so far developed as the corresponding theory in two dimensions, but we shall discuss a few results and a few open problems.

It is classic that Poisson's integral yields directly an expansion of functions which are given harmonic interior to the sphere, continuous in the corresponding closed region.

If the function $u(x, y, z)$ is harmonic interior to a sphere and continuous in the corresponding closed region, then interior to the sphere $u(x, y, z)$ can be expanded in a series of harmonic polynomials in $(x, y, z)$, the series converging uniformly in any closed region interior to the sphere.

This theorem will be used in proving the analog of the corollary (\$2) to Runge's theorem. This analog has already been proved by Bergmann [1], but only for the case of convex regions. The method we shall use is closely related to that of Bergmann, and both methods are intimately related to that of Runge.

If the function $u(x, y, z)$ is harmonic in a closed region $S$ bounded by a simple closed (limited) surface, then in that closed region the function $u(x, y, z)$ can be approximated uni- 
formly as closely as desired by a harmonic polynomial in $(x, y, z)$.

We shall find it convenient to have for reference the following lemma.

LEMmA. Let the two points $\left(x_{0}, y_{0}, z_{0}\right)$ and $\left(x^{\prime}, y^{\prime}, z^{\prime}\right)$ and the simple polygonal curve $C$ joining them lie exterior to the closed point set $S$. If the function $u(x, y, z)$ can be uniformly approximated in $S$ as closely as desired by a harmonic function whose only singularity lies in $\left(x_{0}, y_{0}, z_{0}\right)$, then this function $u(x, y, z)$ can likewise be uniformly approximated in $S$ as closely as desired by a harmonic function whose only singularity lies in $\left(x^{\prime}, y^{\prime}, z^{\prime}\right)$ and which is rational except for the factor $\left[\left(x-x^{\prime}\right)^{2}+\right.$ $\left.\left(y-y^{\prime}\right)^{2}+\left(z-z^{\prime}\right)^{2}\right]^{-1 / 2}$.

We give the proof of this lemma for the case that both $\left(x_{0}, y_{0}, z_{0}\right)$ and $\left(x^{\prime}, y^{\prime}, z^{\prime}\right)$ are finite points, but that is simply a matter of convenience. The proof holds with only obvious changes even if $\left(x^{\prime}, y^{\prime}, z^{\prime}\right)$ is the point at infinity, ${ }^{*}$ and it is in this latter form that the lemma will be applied later. When $\left(x^{\prime}, y^{\prime}, z^{\prime}\right)$ is the point at infinity, the approximating functions are polynomials in $(x, y, z)$, and the factor $\left[\left(x-x^{\prime}\right)^{2}\right.$ $\left.+\left(y-y^{\prime}\right)^{2}+\left(z-z^{\prime}\right)^{2}\right]^{-1 / 2}$ does not enter.

Construct a finite sequence of spheres $S_{0}, S_{1}, \cdots, S_{n}$ extending from $\left(x_{0}, y_{0}, z_{0}\right)$ to $\left(x^{\prime}, y^{\prime}, z^{\prime}\right)$, so that no sphere contains in its interior a point of $S$, but so that successive spheres of the sequence have a region common to them. The point $\left(x_{0}, y_{0}, z_{0}\right)$ shall lie interior to $S_{0}$ and so shall another particular point $\left(x_{1}, y_{1}, z_{1}\right)$ of $C$. The sphere $S_{1}$ shall contain $\left(x_{1}, y_{1}, z_{1}\right)$ in its interior and likewise another particular point $\left(x_{2}, y_{2}, z_{2}\right)$ which lies on $C$ between $\left(x_{1}, y_{1}, z_{1}\right)$ and $\left(x^{\prime}, y^{\prime}, z^{\prime}\right)$. We proceed in this way to construct $S_{2}, S_{3}, \cdots$, $S_{n-1}$, finally arriving at the sphere $S_{n}$ which contains in its interior $\left(x_{n}, y_{n}, z_{n}\right)$ and $\left(x^{\prime}, y^{\prime}, z^{\prime}\right)$. The construction of these spheres is surely possible, for it is no loss of generality to assume that $C$ is composed of merely a finite number of line

* It is to be remembered that vanishing at infinity is a necessary condition that a function should be harmonic there. 
segments and has the length $l$. Assume likewise that the nearest distance from $C$ to a point of $S$ is $\delta$. Choose the sequence of points $\left(x_{0}, y_{0}, z_{0}\right),\left(x_{1}, y_{1}, z_{1}\right), \cdots,\left(x_{n}, y_{n}, z_{n}\right)$, $\left(x^{\prime}, y^{\prime}, z^{\prime}\right)$ on $C$ such that the greatest distance between two successive points is less than $\delta / 2$. The sphere $S_{i}$ may be taken as the sphere with center $\left(x_{i}, y_{i}, z_{i}\right)$ and diameter $\delta$.

Let an arbitrary positive $\epsilon$ be given. We choose the harmonic function $r_{0}(x, y, z)$, whose only singularity lies in $\left(x_{0}, y_{0}, z_{0}\right)$, such that we have

$$
\left|u(x, y, z)-r_{0}(x, y, z)\right|<\frac{\epsilon}{n+2}, \quad(x, y, z) \text { in } S .
$$

This choice is possible by hypothesis. We next choose the harmonic function $r_{1}(x, y, z)$, whose only singularity lies in $\left(x_{1}, y_{1}, z_{1}\right)$, rational except for the factor $\left[\left(x-x_{1}\right)^{2}+\left(y-y_{1}\right)^{2}+\right.$ $\left.\left(z-z_{1}\right)^{2}\right]^{-1 / 2}$, such that we have

$$
\left|r_{0}(x, y, z)-r_{1}(x, y, z)\right|<\frac{\epsilon}{n+2}, \quad(x, y, z) \text { in } S .
$$

We prove by means of an inversion in the unit sphere whose center is $\left(x_{1}, y_{1}, z_{1}\right)$ that such choice is possible:

$$
\begin{aligned}
\xi & =\frac{x-x_{1}}{r^{2}}, \quad \eta=\frac{y-y_{1}}{r^{2}}, \quad \zeta=\frac{z-z_{1}}{r^{2}} \\
r^{2} & =\frac{1}{\rho^{2}}=\left(x-x_{1}\right)^{2}+\left(y-y_{1}\right)^{2}+\left(z-z_{1}\right)^{2} .
\end{aligned}
$$

The function $\rho_{0}(\xi, \eta, \zeta)=(1 / \rho) r_{0}(x, y, z)$, where $\xi, \eta, \zeta, x$, $y, z$ are connected by the relations (17), is a harmonic function of $(\xi, \eta, \zeta)$ on and within $S_{0}^{\prime}$, the transform of the sphere $S_{0}$ under the inversion. Then by the theorem already stated, the function $r^{\prime}(\xi, \eta, \zeta)$, a polynomial in $(\xi, \eta, \zeta)$, can be determined so that we have $\left|\frac{1}{\rho} r_{0}(x, y, z)-r^{\prime}(\xi, \eta, \zeta)\right|<\frac{\epsilon}{(n+2) d},(\xi, \eta, \zeta)$ interior to $S_{0}^{\prime}$, where $d$ is the distance from $\left(x_{1}, y_{1}, z_{1}\right)$ to the farthest point of $S_{0}^{\prime}$. This inequality implies the inequality 
$\left|r_{0}(x, y, z)-\rho r^{\prime}(\xi, \eta, \zeta)\right|<\frac{\epsilon}{n+2}, \quad(x, y, z)$ exterior to $S_{0}$

But $(1 / r) r^{\prime}(\xi, \eta, \zeta)$ is a harmonic function of $(x, y, z)$, rational except for the factor $1 / r$ and with its only singularity in the point $\left(x_{1}, y_{1}, z_{1}\right)$; hence its equal $\rho r^{\prime}(\xi, \eta, \zeta)$ may be identified with the desired function $r_{1}(x, y, z)$ and yields (16).

This same process can now be continued, and yields the additional inequalities

$$
\begin{array}{ccc}
\left|r_{1}(x, y, z)-r_{2}(x, y, z)\right|<\frac{\epsilon}{n+2}, & & (x, y, z) \text { in } S, \\
\cdot & \cdot & \cdot \\
\left|r_{n}(x, y, z)-r_{n+1}(x, y, z)\right|<\frac{\epsilon}{n+2}, & & (x, y, z) \text { in } S .
\end{array}
$$

Hence we have finally

$$
\left|u(x, y, z)-r_{n+1}(x, y, z)\right|<\epsilon, \quad(x, y, z) \text { in } S,
$$

where $r_{n+1}(x, y, z)$ represents a harmonic function whose only singularity lies in $\left(x^{\prime}, y^{\prime}, z^{\prime}\right)$ and is, except for the factor $\left[\left(x-x^{\prime}\right)^{2}+\left(y-y^{\prime}\right)^{2}+\left(z-z^{\prime}\right)^{2}\right]^{-1 / 2}, \quad$ rational in $(x, y, z)$. That is, the lemma is established.

We need a second lemma in the proof. For the sake of simplicity we state and prove the lemma for integrals of a function of a single variable, but the proof holds without essential change for multiple integrals, and to double integrals we shall apply the lemma. Likewise in the proof we consider merely a single parameter $\alpha$, but no difficulty is introduced by the appearance of several, and several parameters appear in the application we shall make.

Lemma. Let $f(x, \alpha)$ be a continuous real function of the arguments for $a \leqq x \leqq b, \alpha_{1} \leqq \alpha \leqq \alpha_{2}$. If an arbitrary positive $\delta$ be given, then there exists $\delta^{\prime}$ so that the inequality

$$
\begin{gathered}
\left|\int_{a}^{b} f(x, \alpha) d x-\sum_{i=1}^{n} f\left(\xi_{i}, \alpha\right)\left(x_{i}-x_{i-1}\right)\right| \leqq \delta, \\
a=x_{0} \leqq x_{1} \leqq \cdots \leqq x_{n}=b, \quad x_{i-1} \leqq \xi_{i} \leqq x_{i},
\end{gathered}
$$


holds uniformly for all $\alpha, \alpha_{1} \leqq \alpha \leqq \alpha_{2}$, provided merely that we have $\left|x_{i}-x_{i-1}\right| \leqq \delta^{\prime}$.

By the mean value theorem for integrals, we can write

$$
\int_{a}^{b} f(x, \alpha) d x=\sum_{i=1}^{n} f\left(\eta_{i}, \alpha\right)\left(x_{i}-x_{i-1}\right)
$$

where $x_{i-1} \leqq \eta_{i} \leqq x_{i}$, and where $\eta_{i}$ depends on $\alpha$. Let us choose $\delta^{\prime}$ so small that the inequality $\left|\zeta_{i}-\zeta_{i}^{\prime}\right| \leqq \delta^{\prime}$ implies

$$
\left|f\left(\zeta_{i}, \alpha\right)-f\left(\zeta_{i}^{\prime}, \alpha\right)\right| \leqq \delta /(b-a)
$$

uniformly for all $\alpha, \alpha_{1} \leqq \alpha \leqq \alpha_{2}$; such a $\delta^{\prime}$ exists by the uniform continuity of $f(x, \alpha)$. Then under the hypothesis $\left|x_{i}-x_{i-1}\right|$ $\leqq \delta^{\prime}$, we have also $\left|\xi_{i}-\eta_{i}\right| \leqq \delta^{\prime}$, from which follows the inequality

$$
\left|\sum_{i=1}^{n} f\left(\eta_{i}, \alpha\right)\left(x_{i}-x_{i-1}\right)-\sum_{i=1}^{n} f\left(\xi_{i}, \alpha\right)\left(x_{i}-x_{i-1}\right)\right| \leqq \delta,
$$

which is precisely (18).

We are now in a position to prove the theorem. Denote by $\sigma$ a simple closed surface consisting entirely of a finite number of portions of planes parallel to the coordinate planes, which contains $S$ in its interior, and on and within which the given function $u(x, y, z)$ is harmonic. The value of $u(x, y, z)$ in $S$ is given by Green's integral

$$
u(x, y, z)=\frac{1}{4 \pi} \int_{\sigma} \int\left[\frac{1}{r} \frac{\partial u}{\partial n}-u \frac{\partial\left(\frac{1}{r}\right)}{\partial n}\right] d \sigma,
$$

where $n$ represents the exterior normal. Let an arbitrary positive $\epsilon$ be given. We divide $\sigma$ into a finite number $n$ of pieces $\sigma_{i}$, so that each piece consists only of a portion of a plane parallel to one of the coordinate planes, so that each piece $\sigma_{i}$ lies interior to a sphere $S_{i}$ which contains on or within it no point of $S$, and so that we have 


$$
\left|\frac{1}{4 \pi} \int_{\sigma} \int \frac{1}{r} \frac{\partial u}{\partial n} d \sigma-\frac{1}{4 \pi} \sum_{i=1}^{n} \frac{1}{r_{i}}\left(\frac{\partial u}{\partial n}\right)_{i} \Delta_{i} \sigma\right| \leqq \frac{\epsilon}{4},
$$

$$
\left|\frac{1}{4 \pi} \int_{\sigma} \int u \frac{\partial(1 / r)}{\partial n} d \sigma-\frac{1}{4 \pi} \sum_{i=1}^{n} u_{i}\left[\frac{\partial(1 / r)}{\partial n}\right]_{i} \Delta_{i} \sigma\right| \leqq \frac{\epsilon}{4},
$$

where $r_{i}$ is the distance from $(x, y, z)$ to a particular but arbitrary point $P_{i}$ of $\sigma_{i}$, where $(\partial u / \partial n)_{i}$ is the normal derivative of $u(x, y, z)$ at $P_{i}$, where $\Delta_{i} \sigma$ is the area of $\sigma_{i}$, where $u_{i}$ is the value of $u(x, y, z)$ at $P_{i}$, and where $[\partial(1 / r) / \partial n]_{i}$ is the value of $\partial(1 / r) / \partial n$ at $P_{i}$. The last expression may also be written as the value at $P_{i}$ of a partial derivative of $1 / r$ with respect not to $(x, y, z)$ but to running coordinates on $\sigma$, and this latter form serves better to indicate that we are dealing with a harmonic function of $(x, y, z)$.

Each term of the sums in (20) is a function of $(x, y, z)$ harmonic not merely in the closed region $S$ but throughout the exterior of the corresponding sphere $S_{i}$, even at infinity. It is immediately seen by inversion with $P_{i}$ as center of inversion that in the $(x, y, z)$-space each of these terms can be approximated by a harmonic function of $(x, y, z)$ whose only singularity lies in $P_{i}$ and which is rational except for the factor $1 / r_{i}$, where $r_{i}$ indicates distance measured from $P_{i}$. The approximation can be made uniformly as closely as desired in the exterior of the sphere $S_{i}$, and hence uniformly as closely as desired in the closed region $S$. But each point $P_{i}$ can be joined to the point at infinity by a Jordan curve which does not meet $S$. Then by the first lemma we can approximate each term of the sums in (20) as closely as desired uniformly in the closed region $S$ by a harmonic polynomial in $(x, y, z)$. That is, we can determine harmonic polynomials $p_{1}(x, y, z)$ and $p_{2}(x, y, z)$ such that we have

$$
\begin{aligned}
& \left|\frac{1}{4 \pi} \sum_{i=1}^{n} \frac{1}{r_{i}}\left(\frac{\partial u}{\partial n}\right)_{i} \Delta_{i} \sigma-p_{1}(x, y, z)\right| \leqq \frac{\epsilon}{4},(x, y, z) \text { in } S, \\
& \left|\frac{1}{4 \pi} \sum_{i=1}^{n} u_{i}\left(\frac{\partial(1 / r)}{\partial n}\right)_{i} \Delta_{i} \sigma-p_{2}(x, y, z)\right| \leqq \frac{\epsilon}{4},(x, y, z) \text { in } S .
\end{aligned}
$$


It follows immediately that we have

(22) $\left|u(x, y, z)-\left[p_{1}(x, y, z)-p_{2}(x, y, z)\right]\right| \leqq \epsilon,(x, y, z)$ in $S$, and the theorem is proved.

The present proof has the advantage over the proof of $\$ 2$ of holding (with only minor modifications) in two, three, or $n$ dimensions, and since it does not involve the theory of functions of a complex variable, has the additional advantage of being more satisfactory from the standpoint of the purist. This remark applies also to the results which immediately follow.

Another remark is of interest. If the point $(x, y, z)$ is exterior to the closed surface $\sigma$, the integral (19) has the value zero, where $r$ still indicates distance from $(x, y, z)$ to an arbitrary point of $\sigma$. Indeed it is a general theorem that if the functions $u$ and $v$ are both harmonic on and within $\sigma$, then we have

$$
\int_{\sigma} \int\left[v \frac{\partial u}{\partial n}-u \frac{\partial v}{\partial n}\right] d \sigma=0 \text {. }
$$

Thus if the function $u(x, y, z)$ is given harmonic on a point set $S$ composed of the closed interiors of two non-intersecting simple closed surfaces, two new non-intersecting simple closed surfaces $\sigma_{1}$ and $\sigma_{2}$ can be constructed which consist entirely of a finite number of portions of planes parallel to the coordinate planes, such that each point of $S$ lies interior either to $\sigma_{1}$ or $\sigma_{2}$, and such that $u(x, y, z)$ is harmonic on and within both $\sigma_{1}$ and $\sigma_{2}$. Then formulas (19), (20), (21), (22) are valid without any change whatever, if we set $\sigma=\sigma_{1}+\sigma_{2}$. A similar fact holds if $S$ falls into $n$ distinct parts. In fact we can prove the following theorem.

Let $S$ be an arbitrary closed limited point set whose complsmentary set (with respect to the entire space) is connected. Then if $u(x, y, z)$ is harmonic on $S$, the function $u(x, y, z)$ can be uniformly approximated on $S$ as closely as desired by a harmonic polynomial in $(x, y, z)$.

There exists a point set $\sigma$ consisting of a finite number of mutually exclusive closed limited simply-connected regions 
$\sigma_{1}, \sigma_{2}, \cdots, \sigma_{n}$ containing all points of $S$ in their interiors, the individual regions bounded entirely by a finite number of portions of planes parallel to the coordinate planes, and such that $u(x, y, z)$ is harmonic on the entire set $\sigma_{1}+\sigma_{2}+\cdots$ $+\sigma_{n}$. The previous method applies without change and the theorem follows.

The following application corresponds to the theorem of Runge; the proof is omitted.

Let $C$ be a point set composed of a finite or infinite number of mutually exclusive simply-connected regions, none of which contains the point at infinity in its interior. If the function $u(x, y, z)$ is harmonic interior to each of these regions, then $u(x, y, z)$ can be expanded on $C$ in a series of harmonic polynomials in $(x, y, z)$, and the series converges uniformly on any closed point set contained in $C$.

We add one further result on general approximation. A closed region $S$ is said to be convex with respect to the interior point $P$ if $S$ is bounded by a surface which is cut in a single point by each ray through $P$. A region which is convex in the usual sense of the word is convex with respect to each of its interior points.

If the region $S$ is a limited closed region convex with respect to some interior point $P$, then an arbitrary function $u(x, y, z)$, harmonic interior to $S$ and continuous in the closed region, can be approximated as closely as desired uniformly in the closed region $S$ by a harmonic polynomial in $(x, y, z)$.

Choose $P$ as origin of coordinates, which involves no loss of generality, and consider the transformation

$$
x^{\prime}=\rho x, \quad y^{\prime}=\rho y, \quad z^{\prime}=\rho z, \quad \rho>1,
$$

which transforms $S$ into a region $S^{\prime}$ which contains $S$ in its interior. The function $u(x, y, z)$ is transformed into a new function

$$
u^{\prime}(x, y, z)=u\left(\frac{x}{\rho}, \frac{y}{\rho}, \frac{z}{\rho}\right)
$$

defined throughout $S^{\prime}$. It follows from the uniform contin- 
uity of $u(x, y, z)$ in $S$ that if $\epsilon>0$ be given, then $\rho$ can be chosen so near to unity that we have

$$
\left|u^{\prime}(x, y, z)-u(x, y, z)\right|<\frac{\epsilon}{2}, \quad(x, y, z) \text { in } S .
$$

For $u^{\prime}(x, y, z)-u(x, y, z)$ is the same as $u(x / \rho, y / \rho, z / \rho)$ $-u(x, y, z)$, which becomes uniformly small with $\rho-1$. The function $u^{\prime}(x, y, z)$ is harmonic in the closed region $S$, so there exists a harmonic polynomial $p(x, y, z)$ such that we have

$$
\left|u^{\prime}(x, y, z)-p(x, y, z)\right|<\frac{\epsilon}{2}, \quad(x, y, z) \text { in } S,
$$

whence follows

$$
|u(x, y, z)-p(x, y, z)|<\epsilon, \quad(x, y, z) \text { in } S,
$$

and the theorem is established.*

It will be noticed that the study of the approximation to an arbitrary function in the plane is fairly well developed, while for space the study is only begun. In particular the general question as to when a function harmonic interior to a threedimensional region and continuous in the closed region (or more generally, defined on a given closed point set) can be uniformly approximated in that closed region (or on that point set) as closely as desired by a harmonic polynomial, seems to be well worth investigating. There is an essential difference in methods and results for two and for three dimensions, for in the latter case the Dirichlet problem does not always have a solution, even for simply-connected regions. Methods of solution of the problem of approximation in three dimensions would presumably depend on the study of the solution of the Dirichlet problem for variable regions, and the result would presumably depend to some extent on whether the region is normal [compare Kellogg, 1].

* The corresponding theorem for analytic functions of a complex variable was given independently by Hilb and Szász, Encyklopädie der mathematischen Wissenschaften, vol. 2, C, II, p. 1276, and by Walsh, Transactions of this Society, vol. 26 (1924), pp. 155-170; p. 168, footnote. 
We mention other problems for three or more dimensions: the study of harmonic polynomials belonging to a general region*, the degree of approximation to functions by harmonic polynomials, the question of interpolation by harmonic polynomials, approximation by harmonic rational functions, - - the solution of all of these problems still lies in the future.

\section{REFERENCES AND BIBLIOGRAPHY}

Bergmann, 1. Mathematische Annalen, vol. 86 (1922), pp. 238-271.

2. Mathematische Annalen, vol. 96(1927), pp. 248-263.

Bochner, 1. Mathematische Zeitschrift, vol. 14 (1922), pp. 180-207.

Carleman, 1. Arkiv för Matematik, Astronomi och Fysik, vol. 17 (192223).

Faber, 1. Mathematische Annalen, vol. 57 (1903), pp. 389-408.

2. Journal für Mathematik, vol. 150 (1920), pp. 79-106.

3. Mathematische Annalen, vol. 69 (1910), pp. 372-443.

Fejér, 1. Göttinger Nachrichten, 1918, pp. 319-331.

Haar, 1. Mathematische Annalen, vol. 78 (1918), pp. 294-311.

Hartogs and Rosenthal, 1. Mathematische Annalen, vol. 100 (1928), pp. 212-263.

Jackson, 1. Transactions of this Society, vol. 21 (1920), pp. 321-332.

Kellogg, 1. This Bulletin, vol. 32 (1926), pp. 601-625.

Lavrentieff, 1. Comptes Rendus, vol. 184 (1927), pp. 1634-35.

Lebesgue, 1. Rendiconti di Palermo, vol. 24 (1907), pp. 371-402.

Merriman, 1. On the expansion of harmonic functions in terms of normalorthogonal harmonic polynomials, not yet published. Presented to the Society (New York), April 1928.

Montel, 1. Séries de Polynomes, Paris, 1910.

Osgood, 1. Funktionentheorie, vol. 1, Leipzig, 1912.

2. Annals of Mathematics, (2), vol. 3 (1901-02), pp. 25-34.

Runge, 1. Acta Mathematica, vol. 6 (1885), pp. 229-244.

Szegö, 1. Mathematische Zeitschrift, vol. 9 (1921), pp. 218-270.

de la Vallée-Poussin, 1. L'Approximation des Fonctions, Paris, 1919.

Walsh, 1. Journal für Mathematik, vol. 159 (1928), pp. 197-209.

2. Proceedings of the National Academy of Sciences, vol. 13 (1927), pp. $175-180$.

3. This Bulletin, vol. 33 (1927), pp. 591-598.

4. Transactions of this Society, vol. 30 (1928), pp. 838-847.

5. On approximation by rational functions to an arbitrary function of a complex variable, shortly to appear in the Transactions of this Society.

\section{HARVARD UNIVERSITY}

* For convex regions a treatment is given by Bergmann, 2. 